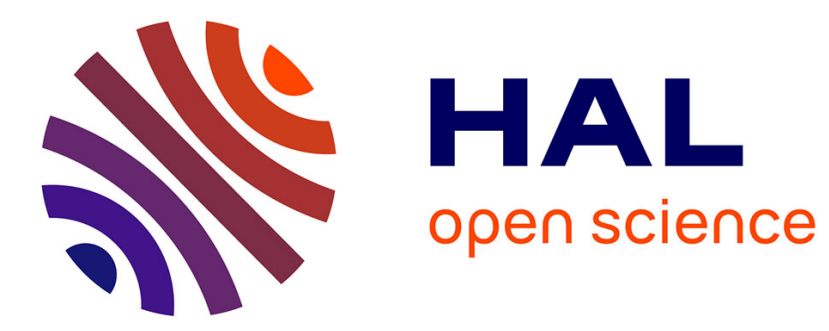

\title{
Navier-Stokes hierarchies of reduced MHD models in Tokamak geometry
}

\author{
Bruno Després, Rémy Sart
}

\section{To cite this version:}

Bruno Després, Rémy Sart. Navier-Stokes hierarchies of reduced MHD models in Tokamak geometry. Journal of Mathematical Fluid Mechanics, 2018, 20, pp.329-357. hal-00796425v2

\section{HAL Id: hal-00796425 \\ https://hal.science/hal-00796425v2}

Submitted on 1 Jul 2013

HAL is a multi-disciplinary open access archive for the deposit and dissemination of scientific research documents, whether they are published or not. The documents may come from teaching and research institutions in France or abroad, or from public or private research centers.
L'archive ouverte pluridisciplinaire HAL, est destinée au dépôt et à la diffusion de documents scientifiques de niveau recherche, publiés ou non, émanant des établissements d'enseignement et de recherche français ou étrangers, des laboratoires publics ou privés. 


\title{
Navier-Stokes hierarchies of reduced MHD models in Tokamak geometry
}

\author{
BRUno DEsPrÉS*, RÉmy SART ${ }^{\star \star}$
}

Reduced MHD models

\begin{abstract}
We study the closure of reduced MHD models. We show how to modify the entropy moment methods to obtain a hierarchy of models of Navier-Stokes type with a correct energy balance. Our procedure is based on the entropy and well adapted to the complicated geometry of the torus. A new correction term due to the geometry is derived, what is important for the stability of the model. We prove the existence of a weak solution to some of these models by similarity with Navier-Stokes theory in potential formulation. We finally obtain a comparison principle between all these models.
\end{abstract}

\section{Introduction}

Reduced magnetohydrodynamics (MHD) systems provide a simplified reduced modeling of the dynamics of magnetic flows in some specific geometrical situations. These models are called reduced because they derive from a simplification (i.e. a reduction) of the system of full MHD (1). This is physically justified to filter non essential magnetosonic waves [25] for flows close to incompressibility. Two major examples are astrophysics [8, 12], and the modeling of Tokamaks in axisymetric geometries for which we refer to the seminal works of Strauss $[44,46,43,47,22,50]$, see also $[2,18]$ and references therein. Despite the interest for applications of this family of models, our understanding is that reduced MHD models have not received enough mathematical attention. The concern in this work is precisely the mathematical structure of such models, having in mind one key domain of application which is the numerical modeling of MHD stability in Tokamaks for which we refer to the recent simulations evoked in $[10,11,15,23]$. See [3] for a more general mathematical and numerical introduction of the topic of MHD stability by different means. Truly agreeing with Krüger and al [26], we consider that energy conservation and closure of the set of equations is important. But contrary to what is usually done in plasma physics where energy balance is checked a posteriori up to some lower order terms [26] and at the price of a very complicated structure of the equations [6], we consider that energy conservation or energy balance must be satisfied a priori. Indeed mathematical stability of a set of non linear PDEs, which is a first step in the direction of proving the well-posedness, is known to be quite sensitive to such a principle: at the discretization level, numerical stability is required to have a control of the stability of the code, and can be an help to design optimal solvers [7]. This is why we consider that having a derivation of reduced MHD models with an exact balance of energy is a fundamental issue. In this direction we will develop a systematic procedure which generates a hierarchy of reduced MHD models with a correct balance of energy and is a way to analyze the complex algebraic structure of such models.

This procedure uses an original extension of the entropy closure method $[9,4,5]$. A systematic application of the entropy closure method to model reduction is to be found in [34]. A more geometrical treatment is in [13], and application to the modeling of uncertainties is in $[38,39]$. We will show that the entropy closure method can be used in toroidal geometries, which is one of the main theoretical contribution of this work ${ }^{1}$. An output

\footnotetext{
* Laboratoire Jacques-Louis Lions, Université Paris VI, 4 place Jussieu, 75015 Paris, despres@ann.jussieu.fr

** Ecole Supérieure d'Ingénieurs Léonard de Vinci, 92916 Paris-La Défense, remy.sart@devinci.fr

1 As an historical comment, we point out that our approach unify two parts of the seminal contributions of Grad which seemed to have independent life in the scientific literature: the first axis is equations of equilibrium states in magnetic
} 
of this approach is a new correction term $Q$ in the equation of the magnetic potential in axisymetric domains. We will indicate why standard reduced MHD models in Tokamak geometry (axisymetric geometry) which do not have this $Q$ may exhibit some linear ill-posedness. The correction term is defined through the solution of a simple linear well-posed variational formulation: this is an interesting property in view of future numerical developments. Another theoretical result attached to our approach is the fact that the dynamics of any model written within this framework gives a lower estimate of the dynamics of the full initial model. This is detailed in theorem 25. This result can be considered as a fundamental justification of the use of reduced models.

To illustrate more precisely the kind of problems and results addressed in this work we start from the system of viscous magnetohydrodynamics

$$
\left\{\begin{array}{l}
\partial_{t} \rho+\nabla \cdot(\rho \mathbf{u})=0 \\
\partial_{t} \mathbf{B}=\nabla \wedge(\mathbf{u} \wedge \mathbf{B})-\eta \nabla \wedge(\nabla \wedge \mathbf{B}) \\
\partial_{t}(\rho \mathbf{u})+\nabla \cdot(\rho \mathbf{u} \otimes \mathbf{u})+\nabla p+\nabla \cdot\left(\frac{|\mathbf{B}|^{2}}{2} \mathbf{I}-\mathbf{B} \otimes \mathbf{B}\right)=\nu \Delta \mathbf{u}, \quad \mathbf{J}=\nabla \wedge \mathbf{B} \\
\partial_{t}(\rho e)+\nabla \cdot(\rho \mathbf{u} e+p \mathbf{u})+\nabla \cdot((\mathbf{u} \wedge \mathbf{B}) \cdot \mathbf{B})=\nu \nabla \cdot(\mathbf{u} \nabla \mathbf{u})+\eta \nabla \cdot(\mathbf{B} \wedge \mathbf{J})
\end{array}\right.
$$

In the equation (1), $\rho$ is the density, $\mathbf{u}$ is the velocity, $\mathbf{B}$ is the magnetic field, $\mathbf{J}$ is the current and $e=$ $\frac{p}{(\gamma-1) \rho}+\frac{1}{2}|\mathbf{u}|^{2}+\frac{1}{2 \rho}|\mathbf{B}|^{2}$ the density of total energy.

The modeling of reduced MHD flows in axisymetric geometry for Tokamaks usually considers representations like

$$
\mathbf{B}=F \nabla \theta+\nabla \psi \wedge \nabla \theta \text { and } \mathbf{u}=\lambda \nabla \theta+\nabla \phi \wedge \nabla \theta,
$$

where the unknowns are the magnetic potential $\psi$, the velocity potential $\phi$ and the parallel velocity $\lambda$. As explained in figure 1, the angular (toroidal) variable $\theta$ of the axisymetric set of variables $(r, \theta, z)$ is deduced from the Cartesian set of variables $(x, y, z)$ : the correspondence is $r=\sqrt{x^{2}+z^{2}}, x=r \cos \theta$ and $z=r \sin \theta$. In (2) $F$ is a forcing term that represents the exterior coils: it is given.

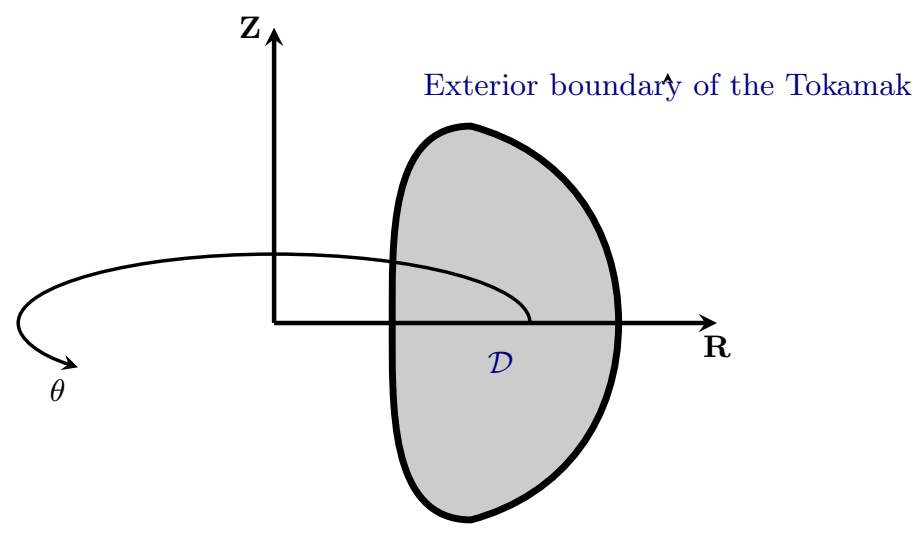

Figure 1. Schematic description of the poloidal section $\mathcal{D}$. The whole Tokamak is the torus $\Omega=\mathcal{D} \times[0,2 \pi]$.

An interesting model in the hierarchy of Navier-Stokes like models that will be constructed is posed in the torus $\Omega=\{(x, z) \in \mathcal{D}$ and $\theta \in[0,2 \pi]\}$ where $\mathcal{D}$ is the poloidal section. It writes in strong form

$$
\left\{\begin{array}{l}
\partial_{t} \psi=\frac{1}{r}[\psi, \phi]+\eta_{\perp} \Delta^{\star} \psi+\eta_{\|} \partial_{\theta}^{2} \psi+\frac{1}{r^{2}} F_{0} \partial_{\theta} \phi+Q \\
\partial_{t} \omega=r\left[\frac{1}{r^{2}} \omega, \phi\right]+r\left[\psi, \frac{1}{r^{2}} \Delta^{\star} \psi\right]+\nu_{\perp} \Delta^{\star} \omega+\nu_{\|} \partial_{\theta}^{2} \omega+\frac{1}{r^{2}} F_{0} \Delta^{\star \star \star} \partial_{\theta} \psi, \\
\omega=\Delta^{\star} \phi
\end{array}\right.
$$

This model does not have parallel velocity. The source term $Q$ is defined by the weak form

$$
\int_{\mathcal{D}} \frac{1}{r}\left(\partial_{r} Q \partial_{r} \tilde{\psi}+\partial_{z} Q \partial_{z} \widetilde{\psi}\right)=2 F_{0} \int_{\mathcal{D}} \frac{1}{r^{4}} \partial_{\theta} \phi \partial_{r} \tilde{\psi}, \quad \forall \tilde{\psi} \in H_{0}^{1}(\mathcal{D}) .
$$

plasmas [22], the famous Grad-Shafranov equation; the second axis is closure methods for the derivation of physically sound non stationary models to compute the dynamics of flows around a given equilibrium [21]. To our knowledge this is the first time that an attempt is made to unify these two fields. 
This model is of Navier-Stokes type in potential formulation. It is representative of the structure of reduced MHD models in toroidal geometry since the non linear terms are some weighted Poisson brackets in the transverse direction. The unknowns are the magnetic potential $\psi$, the velocity potential $\phi$ and the vorticity $\omega$. The density does not show up since this model is incompressible. The radius $r$ everywhere is the non trivial consequence of the curvature of the torus. The Grad-Shafranov diffusion operator is $\Delta^{\star} \psi=\Delta_{\perp} \psi-\frac{1}{r^{2}} \partial_{r} \psi$ while $\Delta^{\star \star \star}$ is another diffusion operator defined in section 4.2. The perpendicular Poisson bracket is $[a, b]=$ $\partial_{r} a \partial_{z} b-\partial_{z} a \partial_{r} b$. The forcing term $F_{0}$ is a constant. We distinguish two viscosity coefficients $\nu_{\perp}$ and $\nu_{\|}$, and two resistivity coefficients $\eta_{\perp}$ and $\eta_{\|}$. The last term is the source term $Q$ which is an original contribution of our approach. We sometimes call it a correction term since it is not present in the standard reduced MHD models. The source term is essential to get a correct energy balance in such a complicated toroidal geometry.

Proposition 1 Neglecting boundary terms, one has the formal energy identity

$$
\begin{gathered}
\frac{1}{2} \frac{d}{d t} \int_{\Omega} \frac{1}{r}\left(\left|\nabla_{r, z} \psi\right|^{2}+\left|\nabla_{r, z} \phi\right|^{2}\right) \\
+\eta_{\perp} \int_{\Omega} \frac{\left|\Delta^{\star} \psi\right|^{2}}{r}+\nu_{\perp} \int_{\Omega} \frac{\left|\Delta^{\star} \phi\right|^{2}}{r}+\eta_{\|} \int_{\Omega} \frac{\left|\partial_{\theta} \nabla_{r, z} \psi\right|^{2}}{r}+\nu_{\|} \frac{\left|\partial_{\theta} \nabla_{r, z} \phi\right|^{2}}{r}=0
\end{gathered}
$$

As a consequence the model (3) is well-posed at least in the weak sense.

Theorem 2 There exists a weak solution to the problem (3-4) equipped with mixed Dirichlet-Neumann boundary condition $\psi=\phi=\frac{\partial \phi}{\partial n}=0$.

The organization of this work is as follows. Next section is devoted to the presentation of our notion of "hyperbolic compatibility", which is an extension of the method of moments $[9,4,13]$. After that we will use this method to obtain new and original reduced MHD models with a correct energy balance, and with the correction term $Q$ in some cases. In the next section we will give a proof of the theorem that explains why the energy balance yields the existence of a weak solution for the viscous problem. This is similar to the theory of Navier-Stokes $[30,31,48]$. Some remarks about the correction term $Q$ show that it may be essential to reach well-posedness in toroidal geometry and for small values of the viscosity and resistivity. Finally we will provide additional material on the linearized stability properties of the abstract procedure of reduction.

\section{General methods to reduce the dimension}

We start from general considerations about hyperbolic systems of conservation laws [27], and more precisely about model reduction of hyperbolic systems of conservation laws.

\subsection{Moment models}

Following $[9,4]$ we consider a hyperbolic system of conservation laws in one dimension

$$
\partial_{t} U+\partial_{x} f(U)=0, \quad x \in \mathbb{R}
$$

where the unknown is $U(x, t) \in \mathbb{R}^{n}$ and the flux is a regular function $f: \mathbb{R}^{n} \rightarrow \mathbb{R}^{n}$. We assume this system is endowed with an entropy-entropy flux pair $U \mapsto(S(U), F(U)) \in \mathbb{R}^{2}$ with the compatibility properties

$$
\nabla S \nabla f=\nabla F \text { and } d^{2} S>0
$$

where $\nabla S, \nabla F \in \mathbb{R}^{n}, \nabla f \in \mathbb{R}^{n \times n}$ and $d^{2} S=d^{2} S^{t}: \mathbb{R}^{n} \rightarrow \mathbb{R}^{n \times n}$ is the Hessian matrix of $S$. For convenience we define the entropy or adjoint variable

$$
V=\nabla S \in \mathbb{R}^{n}
$$

Since $S$ is strictly convex, the transformation $U \mapsto V$ is a diffeomorphism from $\mathbb{R}^{n}$ into (a subset of) $\mathbb{R}^{n}$. A simple type of reduced models are the ones such that some moments are prescribed

$$
\left(U, Z_{i}\right)=a_{i} \in \mathbb{R}, \quad 1 \leq i \leq p \leq n,
$$

where the vectors $Z_{i} \in \mathbb{R}^{n}$ are given, so independent of the time and space variables. The moments (9) are in some sense the new degrees of freedom that one tries to incorporate in (6). Since these constraints have no reason to be invariants of (6) one has to model them, in a stable manner if possible. In this work we rely on a 
procedure that guarantees some transmission of well-posedness from one model to an other. It is based on the minimization of the entropy under constraints (9).

Let us consider the Lagrangian

$$
L(U, \lambda)=S(U)-\sum_{i=1}^{p} \lambda_{i}\left(\left(U, Z_{i}\right)-a_{i}\right)
$$

The optimality conditions write

$$
V-\sum_{i=1}^{p} \lambda_{i} Z_{i}=0
$$

Considering (9) or (10) and assuming the $Z_{i}$ are linearly independent, the unknown $U$ lives in a variety of dimension $p$ and co-dimension $n-p$. The $p$ equations that describe the dynamics of this reduced model are

$$
\partial_{t} a_{i}+\partial_{x}\left(f(U), Z_{i}\right)=0, \quad 1 \leq i \leq p
$$

\subsection{Another formulation}

The previous reduced set of conservation laws (9)-(11) admits a more general formulation that has been developed in a completely different context $[13,38,39]$. It is based on the observation that the variety in which the solution lives is more easily described with the adjoint variable. In particular (10) may be rewritten as a geometrical constraint

$$
V \in K=\operatorname{Span}_{1 \leq i \leq p}\left\{Z_{i}\right\}
$$

Here $K$ is a $d$-dimensional vectorial subspace of $\mathbb{R}^{n}$. It gives hints that, using the entropy variables, model reduction admits a geometrical formulation that we detail now.

The general problem is now to model the constraints $V \in K$ where $K$ is a given closed variety in $\mathbb{R}^{n}$. Considering (12), we will assume that $K$ is an affine hyperplane

$$
\exists V_{0} \in \mathbb{R}^{n} \text { such that } K=V_{0}+\operatorname{Span}_{1 \leq i \leq p}\left\{Z_{i}\right\}
$$

The cone of admissible direction is a vectorial subspace independent of $V$

$$
d K=\operatorname{Span}_{1 \leq i \leq p}\left\{Z_{i}\right\}
$$

The formulation of the reduced system is

$$
\left\{\begin{array}{l}
\partial_{t}\left(U, Z_{i}\right)+\partial_{x}\left(f(U), Z_{i}\right)=0, \quad 1 \leq i \leq p \\
V \in K
\end{array}\right.
$$

There are many ways to prove that this reduced system is hyperbolic. The simplest one consists in remarking that $V-V_{0} \in d K$. Therefore smooth solutions of (14) satisfy $\left(V-V_{0}, \partial_{t} U\right)+\left(V-V_{0}, \partial_{x} f(U)\right)=0$. Making use that $V$ is the entropy variable $(7)$, it can be rearranged as $\partial_{t} \widehat{S}(U)+\partial_{x} \widehat{F}(U)=0$ where

$$
\widehat{S}(U)=S(U)-\left(V_{0}, U\right), \widehat{F}(U)=F(U)-\left(V_{0}, f(U)\right)
$$

Notice that $U \mapsto \widehat{S}(U)$ is a strictly convex functional with respect to $U$ since $d^{2} \widehat{S}=d^{2} S>0$. Up to non essential verifications left to the reader, it shows that $(\widehat{S}(U), \widehat{F}(U))$ is an entropy-entropy flux pair for the reduced system (14). A well known consequence is that (9)-(11) or (10)-(11) is hyperbolic by construction, see $[4,9,13]$. 


\subsection{Hyperbolic compatibility}

At inspection of the type of constraints (2) that we desire to model and analyze, it is nevertheless necessary to extend the previous formulation to functions.

We now consider the following problem, where $\Omega \subset \mathbb{R}^{n}$ is a given open domain and the space variable is denoted as $\mathbf{x} \in \Omega$. For the problems we have in mind, the domain may be a cylinder $\Omega=\mathcal{C}$ or a torus $\Omega=\mathcal{T}$. The flux is $f(U)$ with $f: \mathbb{R}^{n} \rightarrow \mathbb{R}^{n \times d}$. The starting point is the system of conservation laws in dimension $d$

$$
\partial_{t} U+\nabla \cdot f(U)=0 \text {. }
$$

We will denote for convenience $X=\mathcal{C}^{1}(\Omega)$.

Remark 3 The $\mathcal{C}^{1}$ regularity is enough for the weak formulation (17) to make sense. It is not a key ingredient and will be modified in the sequel. For example the strong formulation (52) needs more regularity to make sense. Convenient functional spaces in the context of the Lions-Temam theory will be used in section 4.

Let us consider any affine subspace of $X^{n}$ defined by

$$
\mathcal{K}=V_{0}+d \mathcal{K} \subset X^{n}
$$

where $V_{0} \in X^{n}$ is now a function and $d \mathcal{K}$ is a closed vectorial subspace of $X^{n}$. The dimension of $d \mathcal{K}$ is a priori infinite.

Definition 4 (Hyperbolic compatibility) Neglecting at this stage the boundary conditions, any model that can be written under the form

$$
\left\{\begin{array}{l}
\int_{\Omega}\left[\partial_{t} U+\nabla \cdot f(U), Z\right] d v=0, \forall Z \in d \mathcal{K}, \\
V \in \mathcal{K}
\end{array}\right.
$$

will be said to be hyperbolic compatible.

Remark 5 Boundary conditions, though simple ones, will be reintroduced along this work.

Additional constraints must often be considered to obtain a physically sound set of equations. Such additional constraints can be characterized by the definition of open possibly unbounded domain $\mathcal{P} \subset X^{n}$. Typically any natural physical constraint such as $\rho>0$ or $T>0$ is incorporated in the definition of $\mathcal{P}$. In this case a more precise formulation could be $V \in \mathcal{K} \cap \mathcal{P}$ instead of $V \in \mathcal{K}$. However such considerations add nothing to the main point discussed in this work, at the price of heavier notations. So for the sake of simplicity, we will write the constraint $V \in \mathcal{K}$, having in mind that physically sound unknowns lie in a subset of $\mathcal{K}$. It can also be interesting to recall the distinction that Dirac [16] made between primary constraints and secondary constraints. Primary constraints are naturally respected by the initial model so do not need to be modeled and can be analyzed by almost immediate considerations. The issue is about secondary constraints. For example the constraint $\rho>0$ is a primary constraint. On the other hand $\mathbf{B}=\nabla \psi \wedge \nabla y$ is a secondary constraint. We may make use of this distinction in the following to indicate which constraints are the important ones.

Let us now define the shifted entropy also called a relative entropy $\widehat{S}_{0}(U, \mathbf{x})=S(U)-\left(V_{0}(\mathbf{x}), U\right)$ which is the generalization of (15).

Proposition 6 (A formal entropy or energy identity) A model with hyperbolic compatibility satisfies the identity

$$
\frac{d}{d t} \int_{\Omega} \widehat{S}_{0}(U, \mathbf{x}) d v=\int_{\Omega}\left(\nabla V_{0}(\mathbf{x}): f(U)\right) d v+\text { b.c. }
$$

where b.c. represents integrals on the boundary $\partial \Omega$ and : is the contraction of tensors.

Proof. By definition $\mathcal{K}$ is affine and $V-V_{0} \in d \mathcal{K}$. So (17) yields

$$
\int_{\Omega}\left[\left(\partial_{t} U, V-V_{0}\right)+\left(\nabla \cdot f(U), V-V_{0}\right)\right] d v=0 .
$$

It yields

Integration by parts yields

$$
\int_{\Omega} \partial_{t} \widehat{S}_{0}(U, \mathbf{x}) d v=-\int_{\Omega} \nabla \cdot F(U) d v+\int_{\Omega}\left(\nabla \cdot f(U), V_{0}\right) d v
$$

$$
\int_{\Omega} \partial_{t} \widehat{S}_{0}(U, \mathbf{x}) d v=-\int_{\partial \Omega}(F(U), \mathbf{n}) d \sigma+\int_{\partial \Omega}\left(f(U) V_{0}, \mathbf{n}\right) d \sigma-\int_{\Omega}\left(\nabla V_{0}(x): f(U)\right) d v
$$

The proof is ended. 
Depending on the regularity of $V_{0}$ and the coercivity properties of $S$, the identity (18) may give some informations about the stability of the model.

\section{Application to reduced MHD modeling}

Our purpose is to apply the previous material to define a hierarchy of reduced MHD models with the hyperbolic compatibility. Any model obtained within this formalism will be called an hyperbolic compatible model.

\subsection{Preliminary remarks}

In more comprehensive models all variables display a dependency with respect to $\theta$. Considering the intrinsic complexity of the expansion (2) it is not evident a priori that all kind of reduced MHD models obtained from a reduction of (1) with the representation (2) are endowed with a energy identity. In some cases it has been checked [36] that the energy identity is lost. We first discuss various extensions of (2) in relation with the underlying physical context.

1. A constant forcing term $F=F_{0}$ helps to design a model with an energy identity, see [17,26,25]. Notice moreover that for a constant $F_{0}$ the associated magnetic force is zero since $\nabla \wedge \nabla \theta=0$ : the forcing term is, physically, force-free.

2. It has never been proved that a non constant forcing term generates a reduced model with energy balance: see all references therein this work. A priori, non constant $F$ yields a non force-free magnetic force $\nabla \wedge$ $(F \nabla \theta) \wedge(F \nabla \theta) \neq 0$. This is why a energy balance should be looked for in this case, and not an energy conservation. The theory developed in this work will provide a systematic tool to analyze this situation: see the abstract balance equation (18).

3. In physical situations we are interested in, the flow is nearly incompressible. This is compatible, in some sense, with the perpendicular part of the velocity (2) which is divergence free. But in this case the incompressibility is reached only if the density is constant in space due to $\partial_{t} \rho+\mathbf{u} \cdot \nabla \rho=0$. It corresponds to the basic reduced model (24).

4. The Ansatz in toroidal geometry mostly used in the physical literature $[6,17,25,26,10,11,23,26,40,42,46$, 50] is based on $\mathbf{u}=\mathbf{u}_{\|}+\mathbf{u}_{\perp}$ with

$$
\mathbf{u}_{\perp}=r^{2} \nabla \phi \wedge \nabla \theta
$$

The perpendicular velocity is not divergence free a priori, even if it is nearly divergence free for small curvatures for which the variation of $r$ is negligible in first approximation. The idea behind this choice is some compatibility with the Ohm's law $\mathbf{E}+\mathbf{u} \wedge \mathbf{B}=0$ which is a key ingredient to eliminate the electric field in the set of full Maxwell's equation: $\partial_{t} \mathbf{B}+\nabla \wedge \mathbf{E}=0$ is indeed equivalent to $\partial_{t} \mathbf{B}=\nabla \wedge \mathbf{u} \wedge \mathbf{B}$. Let us now consider a magnetic field under the form $\mathbf{B}=F_{0} \nabla \theta$ and let assume a perpendicular velocity (19) with a potential invariant in the toroidal direction: $\partial_{\theta} \phi=0$. With these hypotheses, one has after simplifications $\left(\nabla \theta=\frac{1}{r} \mathbf{e}_{\theta}\right)$

$$
\mathbf{E}=-r^{2}(\nabla \phi \wedge \nabla \theta) \wedge\left(F_{0} \nabla \theta\right)=F_{0} \nabla \phi
$$

It means the electric field is potential. A restriction is $\partial_{\theta} \phi=0$. This idea is developed in $[17,26,25]$ and references therein.

5. In this work we prefer to develop closure relations that correspond to nearly incompressible regimes. In this direction it has been observed in [15] that incompressibility (that is $\partial_{t} \rho=0$ ) with a non trivial and non constant in space density can be obtained with

$$
\mathbf{u}_{\perp}=\frac{1}{\rho_{0}} \nabla \phi \wedge \nabla \theta, \quad \rho(t=0)=\rho_{0} .
$$

We will develop such a model in our work. The usual Ansatz (19) is formerly recovered as $\rho_{0}=r^{-2}$. This is why there is no contradiction between (19) and (20). We will use this similarity in (51).

6. The material presented in this work can be developped for the modeling of the parallel velocity, as in [45]. One can use $\mathbf{u}_{\|}=\lambda \mathbf{B}_{0}$ where $\mathbf{B}_{0}$ is a given a priori and frozen magnetic field which therefore replaces $\nabla \theta$. For example one can take the magnetic field at initial time. 
7. In some cases it is worthwhile to consider an extended magnetic equation with a source term

$$
\partial_{t} \mathbf{B}=\nabla \wedge(\mathbf{u} \wedge \mathbf{B})-\eta \nabla \wedge(\nabla \wedge \mathbf{B})+\eta \nabla \wedge\left(\nabla \wedge \mathbf{B}_{\text {boot }}\right)
$$

where $\mathbf{B}_{\text {boot }}$ is given magnetic field. In the context of Tokamaks modeling, it generates what is called a bootstrap current. It represents a forcing term that one adds to the induction equation in order to obtain the observed equilibrium. It can be as in the example an external magnetic field, which even if it may seem unrealistic, is essential to obtain physically relevant MHD numerical simulations $[10,32,33,24,19,15]$.

\subsection{The adjoint variable}

The first step is to identify the formulation of full ideal MHD such that $\mathbf{B}$ and $\mathbf{u}$ are components of the adjoint variable, so that relations like $(2)$ can be used to define $\mathcal{K}$. We use the fact that flows in Tokamaks are smooth. Therefore one can use either the energy formulation (1) rewritten for convenience in its non viscous and non resistive version

$$
\left\{\begin{array}{l}
\partial_{t} \rho+\nabla \cdot(\rho \mathbf{u})=0 \\
\partial_{t}(\rho \mathbf{u})+\nabla \cdot(\rho \mathbf{u} \otimes \mathbf{u})+\nabla p+\nabla \cdot\left(\frac{|\mathbf{B}|^{2}}{2} \mathbf{I}-\mathbf{B} \otimes \mathbf{B}\right)=0 \\
\partial_{t} \mathbf{B}-\nabla \wedge(\mathbf{u} \wedge \mathbf{B})=0 \\
\partial_{t}(\rho e)+\nabla \cdot(\rho \mathbf{u} e+p \mathbf{u})+\nabla \cdot((\mathbf{u} \wedge \mathbf{B}) \cdot \mathbf{B})=0
\end{array}\right.
$$

or its absolutely equivalent isentropic formulation

$$
\left\{\begin{array}{l}
\partial_{t} \rho+\nabla \cdot(\rho \mathbf{u})=0 \\
\partial_{t}(\rho \mathbf{u})+\nabla \cdot(\rho \mathbf{u} \otimes \mathbf{u})+\nabla p+\nabla \cdot\left(\frac{|\mathbf{B}|^{2}}{2} \mathbf{I}-\mathbf{B} \otimes \mathbf{B}\right)=0 \\
\partial_{t} \mathbf{B}-\nabla \wedge(\mathbf{u} \wedge \mathbf{B})=0 \\
\partial_{t}(\rho s)+\nabla \cdot(\rho \mathbf{u} s)=0
\end{array}\right.
$$

The equivalence for smooth flows between (21) and (22) is described in many textbooks. It uses the freedivergence $\nabla \cdot \mathbf{B}=0$ which is true for all times since it is true at initial time.

The exact form of the density or entropy equation is not important. For example it can be replaced by the pressure equation $\partial_{t} p+\mathbf{u} \cdot \nabla p+\gamma p \nabla \cdot \mathbf{u}=0$ without changing our results. For the simplicity of mathematical notations, we prefer to use the entropy equation (22).

Next step is to determine the entropy variable. It is a consequence of the fundamental principle of thermodynam$\operatorname{ics} T d s=d \varepsilon+p d \tau$. Defining $e=\varepsilon+\frac{1}{2}|\mathbf{u}|^{2}+\frac{1}{2} \tau|\mathbf{B}|^{2}$, it implies $T d s=d e-\mathbf{u} \cdot d \mathbf{u}-\mathbf{B} \cdot d(\tau \mathbf{B})+\left(p+|\mathbf{B}|^{2}\right) d \tau$, and so $T d(\rho s)=d(\rho e)-\mathbf{u} \cdot d(\rho \mathbf{u})-\mathbf{B} \cdot d \mathbf{B}+\mu d \rho$ where $\mu=T s-e+|\mathbf{u}|^{2}+\tau|\mathbf{B}|^{2}-p \tau$ is the Gibbs thermodynamical potential. This differential relation can be summarized as follows.

Proposition 7 The convex entropy of system (21) is $S=-\rho s$ with

$$
U=(\rho, \rho \mathbf{u}, \mathbf{B}, \rho e) \text { and adjoint variable } V=-\frac{1}{T}(\mu,-\mathbf{u},-\mathbf{B}, 1)
$$

The convex entropy of system (22) is $S=\rho e$ with

$$
U=(\rho, \rho \mathbf{u}, \mathbf{B}, \rho s) \text { and adjoint variable } V=(-\mu, \mathbf{u}, \mathbf{B}, T) .
$$

By comparison with (2) it is immediate to realize that (2) can be interpreted as a constraint for the isentropic system (22) and not for (21) because of the $\frac{1}{T}$ term which spoils the structure. That is why we will now consider only (22) in the following.

\subsection{Derivation of the fundamental model in a cylinder}

We now desire to explain how to recover the standard reduced model in planar geometry through the definition of an appropriate set $\mathcal{K}$. The domain is a cylinder $\Omega=\mathcal{C}=\{(x, y, z) ;(x, z) \in \mathcal{D}, y \in \mathbb{R}\}$ where $\mathcal{D}$ is a smooth bounded domain $\mathcal{D} \subset \mathbb{R}^{2}$. The integration measure is $d v=d x d y d z$. 
Proposition 8 Let $F_{0} \in \mathbb{R}$ be a constant and

$$
Y=\left\{(a, \phi, \psi, b) \in X^{4} ; \partial_{y} \phi=\partial_{y} \psi=0\right\} \subset X^{4} .
$$

Consider the reduced model deduced from the set $\mathcal{K} \subset X^{8}$

$$
\mathcal{K}=\left(0,0, F_{0} \nabla y, 0\right)+\operatorname{Span}_{(a, \phi, \psi, b) \in Y}\{a, \nabla \phi \wedge \nabla y, \nabla \psi \wedge \nabla y, b\} .
$$

Assume the initial data is $\rho \equiv 1$ and $T \equiv 1$. Assume boundary data for the magnetic potential and for the velocity potential: $\psi=\phi=\partial_{n} \phi=0$ on $\partial \Omega$. The corresponding reduced model written in the transverse $2 D$ domain $\mathcal{D}$ is

$$
\left\{\begin{array}{l}
\partial_{t} \psi=[\psi, \phi] \\
\partial_{t} \omega=[\omega, \phi]+\left[\psi, \Delta_{\perp} \psi\right] \\
\Delta_{\perp} \phi=\omega .
\end{array}\right.
$$

Remark 9 The boundary condition is chosen for its simplicity. It insures that $\mathbf{u}=0$ on the wall, which yields the total mass in the domain is constant.

Proof. One has $d \mathcal{K}=\operatorname{Span}_{Y}\{a, \nabla \phi \wedge \nabla y, \nabla \psi \wedge \nabla y, b\} \subset X^{8}$. We split the proof in three parts. The first part is the thermodynamic part which is easy since $\rho=1$ and $T=1$ are primary constraints. The second part is the velocity part which is a secondary constraint: the manipulations are somewhat identical to the ones performed in the usual physical derivation. The third part is for the magnetic field, it is also a secondary constraint: the result is the usual one even if the method is slightly different for reasons explained below. For convenience we use $X_{0}=\mathcal{D}_{0}^{\infty}(\Omega) \subset X$ the set of smooth function with compact support in $\Omega$.

Thermodynamic part: Consider the test vector $Z=(a, 0,0,0) \in d \mathcal{K}$ for all $a \in X_{0}$. One deduces from (17) that

$$
\int_{\mathcal{C}}\left(\partial_{t} \rho+\nabla \cdot(\rho \mathbf{u})\right) a d v=0, \quad \forall a \in X_{0}
$$

It yields: $\partial_{t} \rho+\nabla \cdot(\rho \mathbf{u})=0$ for all $\mathbf{x} \in \Omega$. The same algebra holds for the last equation, that is $\partial_{t}(\rho s)+\nabla$. $(\rho \mathbf{u} s)=0$. By construction $\mathbf{u}=\nabla \wedge(\phi \nabla y)$ is divergence free: $\nabla \cdot \mathbf{u}=0$. Therefore $\rho$ and $T$ are uniformly equal to 1 .

Velocity part: The velocity equation can be written as

$$
\int_{\mathcal{C}}\left(\partial_{t}(\rho \mathbf{u})+\nabla \cdot(\rho \mathbf{u} \otimes \mathbf{u})+\nabla p+\nabla \cdot\left(\frac{|\mathbf{B}|^{2}}{2} \mathbf{I}-\mathbf{B} \otimes \mathbf{B}\right)\right) \cdot \nabla \wedge(\widetilde{\phi} \nabla y) d v=0
$$

for all test functions $\widetilde{\phi} \in X_{0}$ with $\partial_{y} \widetilde{\psi}=0$. An integration by parts yields

$$
\int_{\mathcal{C}} \widetilde{\phi} \nabla y \cdot \nabla \wedge\left(\partial_{t}(\rho \mathbf{u})+\nabla \cdot(\rho \mathbf{u} \otimes \mathbf{u})-\nabla \cdot(\mathbf{B} \otimes \mathbf{B})+\nabla\left(p+\frac{|\mathbf{B}|^{2}}{2}\right)\right) d v=0 .
$$

Note that

$$
\widehat{\psi}=\nabla y \cdot \nabla \wedge\left(\partial_{t}(\rho \mathbf{u})+\nabla \cdot(\rho \mathbf{u} \otimes \mathbf{u})-\nabla \cdot(\mathbf{B} \otimes \mathbf{B})+\nabla\left(p+\frac{|\mathbf{B}|^{2}}{2}\right)\right)
$$

is such that $\widehat{\psi} \in X$ and $\partial_{y} \widehat{\psi}=0$. Since $\widetilde{\phi}$ is arbitrary in $X_{0}$ (with $\partial_{y} \widetilde{\phi}$ ), evident simplifications and $\nabla \wedge \nabla\left(p+\frac{|\mathbf{B}|^{2}}{2}\right)=0$ yield

$$
\nabla y \cdot\left(\partial_{t}(\nabla \wedge \mathbf{u})+\nabla \wedge \nabla \cdot(\mathbf{u} \otimes \mathbf{u})-\nabla \wedge \nabla \cdot(\mathbf{B} \otimes \mathbf{B})\right)=0, \quad \mathbf{x} \in \Omega
$$

Since by hypothesis $\mathbf{u}=\nabla \wedge(\phi \nabla y)$, then $\mathbf{u}=\left(-\partial_{z} \phi, 0, \partial_{x} \phi\right)$ and $\nabla \wedge \mathbf{u}=-\Delta_{\perp} \phi \mathbf{e}_{y}=-\Delta_{\perp} \phi \nabla y$. So $\nabla y \cdot \partial_{t}(\nabla \wedge \mathbf{u})=-\partial_{t} \Delta_{\perp} \phi$. We compute $\nabla \wedge \nabla \cdot(\mathbf{u} \otimes \mathbf{u})$ as follows

$$
\begin{gathered}
\mathbf{u} \otimes \mathbf{u}=\left(\begin{array}{ccc}
\partial_{z} \phi^{2} & 0 & -\partial_{x} \phi \partial_{z} \phi \\
0 & 0 & 0 \\
-\partial_{x} \phi \partial_{z} \phi & 0 & \partial_{x} \phi^{2}
\end{array}\right), \\
\nabla \cdot(\mathbf{u} \otimes \mathbf{u})=\left(\partial_{x}\left(\partial_{z} \phi^{2}\right)-\partial_{z}\left(\partial_{x} \phi \partial_{z} \phi\right), 0,-\partial_{x}\left(\partial_{x} \phi \partial_{z} \phi\right)+\partial_{z}\left(\partial_{x} \phi^{2}\right)\right)^{t} \\
=\left(\partial_{z} \phi \partial_{x z} \phi-\partial_{x} \phi \partial_{z z} \phi, 0, \partial_{x} \phi \partial_{x z} \phi-\partial_{z} \phi \partial_{x x} \phi\right)^{t},
\end{gathered}
$$


and

$$
\begin{gathered}
\nabla y \cdot \nabla \wedge \nabla \cdot(\mathbf{u} \otimes \mathbf{u})= \\
=\partial_{z}\left(\partial_{z} \phi \partial_{x z} \phi-\partial_{x} \phi \partial_{z z} \phi\right)-\partial_{x}\left(\partial_{x} \phi \partial_{x z} \phi-\partial_{z} \phi \partial_{x x} \phi\right) \\
=-\partial_{x} \phi \partial_{z} \Delta_{\perp} \phi+\partial_{z} \phi \partial_{x} \Delta_{\perp} \phi=-\left[\phi, \Delta_{\perp} \phi\right] .
\end{gathered}
$$

Similarly, since $\mathbf{B}=\nabla \wedge(\psi \nabla y): \nabla y \cdot \nabla \wedge \nabla \cdot(\mathbf{B} \otimes \mathbf{B})=-\left[\psi, \Delta_{\perp} \psi\right]$.

Therefore (25) yields

$$
\partial_{t} \omega=[\omega, \phi]+\left[\psi, \Delta_{\perp} \psi\right], \quad(x, z) \in \mathcal{D},
$$

where $\omega=\Delta_{\perp} \phi$ is the vorticity.

Magnetic part: The magnetic equation yields

$$
\int_{\mathcal{C}}\left(\partial_{t} \mathbf{B}-\nabla \wedge(\mathbf{u} \wedge \mathbf{B})\right) \cdot \nabla \wedge(\widetilde{\psi} \nabla y) d v=0
$$

for all test functions $\widetilde{\psi} \in X_{0}$ such that $\partial_{y} \widetilde{\psi}=0$. It is possible to integrate the equation by part directly. However it is preferable to notice first that $\partial_{t} \mathbf{B}=\partial_{t}(\nabla \psi \wedge \nabla y)=\nabla \wedge\left(\partial_{t} \psi \nabla y\right)$. We obtain the weak formulation

$$
\int_{\mathcal{C}} \nabla \wedge\left(\partial_{t} \psi \nabla y-\mathbf{u} \wedge \mathbf{B}\right) \cdot \nabla \wedge(\widetilde{\psi} \nabla y) d v=0
$$

Next we integrate by part

$$
\int_{\mathcal{C}}\left(\partial_{t} \psi \nabla y-\mathbf{u} \wedge \mathbf{B}\right) \cdot \nabla \wedge \nabla \wedge(\widetilde{\psi} \nabla y) d v=0
$$

Since $\nabla \cdot \widetilde{\psi} \nabla y=0$ by hypothesis, then $\nabla \wedge \nabla \wedge(\widetilde{\psi} \nabla y)=-\Delta \widetilde{\psi} \nabla y=-\Delta_{\perp} \widetilde{\psi} \nabla y$. After simplifications

$$
\int_{\mathcal{C}}\left(\partial_{t} \psi-(\mathbf{u} \wedge \mathbf{B}) \cdot \nabla y\right) \Delta_{\perp} \widetilde{\psi}=0, \quad \forall \widetilde{\psi} \in X_{0}, \partial_{y} \widetilde{\psi}=0
$$

By construction the function $\partial_{t} \psi-(\mathbf{u} \wedge \mathbf{B}) \cdot \nabla y$ is independent of the variable $y$. So we obtain that $\partial_{t} \psi-$ $(\mathbf{u} \wedge \mathbf{B}) \cdot \nabla y$ is in the kernel of $\Delta_{\perp}^{*}=\Delta_{\perp}$. That is

$$
\partial_{t} \psi-(\mathbf{u} \wedge \mathbf{B}) \cdot \nabla y=Q, \quad \Delta_{\perp} Q=0, \quad(x, z) \in \mathcal{D} .
$$

The hypotheses of the theorem assume natural boundary conditions such as $\psi=0$ and $\mathbf{u}=0$ on the boundary of the domain. It yields $Q=0$ on the boundary $\mathcal{D}$. In this case $Q=0$. We obtain the equation

$$
\partial_{t} \psi-(\mathbf{u} \wedge \mathbf{B}) \cdot \nabla y=0 .
$$

Since $\mathbf{u}=\left(-\partial_{z} \phi, 0, \partial_{x} \phi\right)$ and $\mathbf{B}=\left(-\partial_{z} \psi, F_{0}, \partial_{x} \psi\right)$, evident calculations yield $(\mathbf{u} \wedge \mathbf{B}) \cdot \nabla y=[\psi, \phi]$. We finally obtain

$$
\partial_{t} \psi=[\psi, \phi], \quad(x, z) \in \mathcal{D} .
$$

The proof is ended.

Remark 10 The identity (18) can be written, retaining only the variables which makes sense for (23) or equivalently for (24): that is $V_{0}=0$ and $\widehat{S}_{0}=\frac{1}{2}|\mathbf{B}|^{2}+\frac{1}{2}|\mathbf{u}|^{2}$ and $\rho \equiv 1$. At the boundary, it is a mixed of homogeneous Dirichlet and Neumann conditions. The energy identity reads

$$
\int_{\mathcal{D}} \frac{|\nabla \psi|^{2}+|\nabla \psi|^{2}}{2} d x d z=0 .
$$

Therefore the method of hyperbolic compatibility provides effectively a systematic way to design reduced models with the preservation of the total energy. Since our method of construction is general, we are now in position to describe a hierarchy of reduced MHD models in axisymetric configuration, in the context of the modeling of Tokamaks. 


\subsection{Models with toroidal invariance}

The domain is a torus $\Omega=\mathcal{T}=\mathcal{D} \times[0,2 \pi]$ where $\mathcal{D}$ is a smooth bounded domain $\mathcal{D} \subset \mathbb{R}^{2}$. The integration measure in $\mathcal{T}$ is $d v=d x d y d z=2 \pi r d r d \theta d z$. The reduced models written in $\mathcal{D}$ will use the reduced measure of integration $d r d z$.

The local direct orthogonal basis is $\left(\mathbf{e}_{r}, \mathbf{e}_{\theta}, \mathbf{e}_{z}\right)$ with

$$
\mathbf{e}_{r}=\nabla r, \quad \frac{1}{r} \mathbf{e}_{\theta}=\nabla \theta, \quad \mathbf{e}_{z}=\nabla z
$$

It turns into $\nabla \wedge \mathbf{e}_{r}=\nabla \wedge \mathbf{e}_{z}=\nabla \wedge\left(\frac{1}{r} \mathbf{e}_{\theta}\right)=0$. We also notice that $\Delta \theta=0$ because $\theta$ is an harmonic function. The space of functions independent of the toroidal variable is

$$
X_{\perp}=\left\{h \in X, \partial_{\theta} h=0\right\} \subset X .
$$

We also define $Y=X \times X_{\perp}^{2} \times X$.

Our method of presentation is to introduce various effects through convex sets with increasing complexity. We hope that it helps to distinguish between the various phenomena, still having the possibility to identify the related algebra.

3.4.1. A first poloidal model Here we assume $\rho \equiv 1, s \equiv 1$ and consider

$$
\mathcal{K}_{1}=\operatorname{Span}_{(a, \phi, \psi, b) \in Y}(a, \nabla \phi \wedge \nabla \theta, \nabla \psi \wedge \nabla \theta, b) \subset X^{8}
$$

Since the velocity is divergence free, it is sufficient to assume that $\rho=s=1$ at initial time. Therefore the temperature is constant also. This is the reason why density and temperature do not show up in the next model.

Proposition 11 Assume Dirichlet-Neumann boundary data for the magnetic and velocity potentials: $\psi=\phi=$ $\partial_{n} \phi=0$ on $\partial \Omega$. The incompressible and isothermal reduced model in $\mathcal{D}$ deduced from $\mathcal{K}_{1}$ writes in strong form

$$
\left\{\begin{array}{l}
\partial_{t} \psi=\frac{1}{r}[\psi, \phi] \\
\partial_{t} \omega=r\left[\frac{1}{r^{2}} \omega, \phi\right]-r\left[\frac{1}{r^{2}} \Delta^{\star} \psi, \psi\right]
\end{array}\right.
$$

where the vorticity is $\omega=\Delta^{\star} \phi$.

This model is endowed with the formal energy identity

$$
\frac{d}{d t} \int_{\mathcal{D}} \frac{|\nabla \psi|^{2}+|\nabla \phi|^{2}}{2 r} d r d z=0 .
$$

Remark 12 This model is used in [10] for numerical purposes. It is constructed in [15] following the usual physical point of view. See [14] for numerical discretization.

Proof. Starting with (17) related to the MHD model (22), with test functions $Z=(0, \nabla \widetilde{\phi} \wedge \nabla \theta, \nabla \widetilde{\psi} \wedge \nabla \theta, 0) \in$ $d \mathcal{K}$, one writes

$$
\left\{\begin{array}{l}
\int_{\mathcal{T}}\left[\partial_{t} \mathbf{u}+\nabla \cdot(\mathbf{u} \otimes \mathbf{u})+\nabla p+\nabla \cdot\left(\frac{|\mathbf{B}|^{2}}{2} \mathbf{I}-\mathbf{B} \otimes \mathbf{B}\right)\right] \cdot \nabla \widetilde{\phi} \wedge \nabla \theta d v=0, \\
\int_{\mathcal{T}}\left[\partial_{t} \mathbf{B}-\nabla \wedge(\mathbf{u} \wedge \mathbf{B})\right] \cdot \nabla \widetilde{\psi} \wedge \nabla \theta d v=0, \\
\mathbf{u}=\nabla \phi \wedge \nabla \theta, \quad \mathbf{B}=\nabla \psi \wedge \nabla \theta .
\end{array}\right.
$$

To simplify the notations, we will use a subset of test functions, namely $\widetilde{\psi}, \widetilde{\phi} \in X_{0} \cap X_{\perp}$, so the test functions vanish (and also all their derivatives) at the boundary. 
Velocity part: Recalling that $\nabla \widetilde{\phi} \wedge \nabla \theta=\nabla \wedge(\widetilde{\phi} \nabla \theta)$, and integrating by parts, we get for all $\widetilde{\phi} \in X_{0} \cap X_{\perp}$

$$
\int_{\mathcal{T}} \nabla \wedge\left[\partial_{t} \mathbf{u}+\nabla \cdot(\mathbf{u} \otimes \mathbf{u})+\nabla p+\nabla \cdot\left(\frac{|\mathbf{B}|^{2}}{2} \mathbf{I}-\mathbf{B} \otimes \mathbf{B}\right)\right] \cdot \nabla \theta \widetilde{\phi} d v=0 .
$$

One notices that $\nabla \wedge \nabla\left(p+\frac{|B|^{2}}{2}\right)=0$. Since all functions under the integral are independent of the variable $\theta$, and since this integral equation is satisfied for all arbitrary test function $\widetilde{\phi}$, we deduce

$$
\nabla \wedge\left[\partial_{t} \mathbf{u}+\nabla \cdot(\mathbf{u} \otimes \mathbf{u})-\nabla \cdot(\mathbf{B} \otimes \mathbf{B})\right] \cdot e_{\theta}=0, \quad \mathbf{x} \in \mathcal{T} .
$$

Since this function does not depend on the toroidal variable, the same equality can be written equivalently in the reduced domain $(r, z) \in \mathcal{D}$.

We write $\mathbf{u}=\nabla \phi \wedge \nabla \theta=\left(-\frac{1}{r} \partial_{z} \phi, 0, \frac{1}{r} \partial_{r} \phi\right)$. We note $\alpha=-\frac{1}{r} \partial_{z} \phi$ and $\beta=\frac{1}{r} \partial_{r} \phi$ so that $\mathbf{u}=(\alpha, 0, \beta)$. Then, we calculate

$$
\begin{gathered}
(\nabla \wedge \mathbf{u}) \cdot e_{\theta}=\partial_{z}\left(-\frac{1}{r} \partial_{z} \phi\right)-\partial_{r}\left(\frac{1}{r} \partial_{r} \phi\right) \\
=-\frac{1}{r} \partial_{z}^{2} \phi-\frac{1}{r} \partial_{r}^{2} \phi+\frac{1}{r^{2}} \partial_{r} \phi=-\frac{1}{r} \Delta_{\perp} \phi+\frac{1}{r^{2}} \partial_{r} \phi .
\end{gathered}
$$

Since the Grad-Shafranov operator is defined by $\Delta^{\star} \phi=\Delta_{\perp} \phi-\frac{1}{r^{2}} \partial_{r} \phi$, one obtains

$$
\nabla \wedge\left(\partial_{t} \mathbf{u}\right) \cdot e_{\theta}=-\frac{1}{r} \partial_{t} \Delta^{\star} \phi
$$

Next, remarking that $\nabla \cdot(\mathbf{u} \otimes \mathbf{u})=\mathbf{u} \cdot \nabla \mathbf{u}($ since $\nabla \cdot \mathbf{u}=0)$, one has that $\nabla \cdot(\mathbf{u} \otimes \mathbf{u})=\left(\alpha \partial_{r} \alpha+\beta \partial_{z} \alpha, 0, \alpha \partial_{r} \beta+\beta \partial_{z} \beta\right)^{t}$ and

$$
\begin{array}{r}
\nabla \wedge \nabla \cdot(\mathbf{u} \otimes \mathbf{u}) \cdot e_{\theta}=\partial_{z}\left(\alpha \partial_{r} \alpha+\beta \partial_{z} \alpha\right)-\partial_{r}\left(\alpha \partial_{r} \beta+\beta \partial_{z} \beta\right) \\
=\alpha \partial_{r}\left(\partial_{z} \alpha-\partial_{r} \beta\right)+\beta \partial_{z}\left(\partial_{z} \alpha-\partial_{r} \beta\right)+\left(\partial_{z} \alpha-\partial_{r} \beta\right)\left(\partial_{r} \alpha+\partial_{z} \beta\right) .
\end{array}
$$

Since $\partial_{z} \alpha-\partial_{r} \beta=\partial_{z}\left(-\frac{1}{r} \partial_{z} \phi\right)-\partial_{r}\left(\frac{1}{r} \partial_{r} \phi\right)=-\frac{1}{r} \partial_{z}^{2} \phi-\frac{1}{r} \partial_{r}^{2} \phi+\frac{1}{r^{2}} \partial_{r} \phi=-\frac{1}{r} \Delta^{\star} \phi$ and $\partial_{r} \alpha+\partial_{z} \beta=\partial_{r}\left(-\frac{1}{r} \partial_{z} \phi\right)+$ $\partial_{z}\left(\frac{1}{r} \partial_{r} \phi\right)=-\frac{1}{r} \partial_{r} \partial_{z} \phi+\frac{1}{r^{2}} \partial_{z} \phi+\frac{1}{r} \partial_{z} \partial_{r} \phi=\frac{1}{r^{2}} \partial_{z} \phi$, we finally obtain

$$
\begin{aligned}
\nabla \wedge(\mathbf{u} \otimes \mathbf{u}) \cdot e_{\theta} & =-\frac{1}{r} \partial_{z} \phi \partial_{r}\left(-\frac{1}{r} \Delta^{\star} \phi\right)+\frac{1}{r} \partial_{r} \phi \partial_{z}\left(-\frac{1}{r} \Delta^{\star} \phi\right)-\frac{1}{r^{3}} \Delta^{\star} \phi \partial_{z} \phi \\
& =\partial_{z} \phi \partial_{r}\left(\frac{1}{r^{2}} \Delta^{\star} \phi\right)-\partial_{r} \phi \partial_{z}\left(\frac{1}{r^{2}} \Delta^{\star} \phi\right) \\
& =\left[\frac{1}{r^{2}} \Delta^{\star} \phi, \phi\right]
\end{aligned}
$$

Similarly, we deal with the magnetic term and get

$$
\nabla \wedge(\mathbf{B} \otimes \mathbf{B}) \cdot e_{\theta}=\left[\frac{1}{r^{2}} \Delta^{\star} \psi, \psi\right]
$$

and finally write the final form of the equation (32) including the relations (33), (34) and (35):

$$
-\frac{1}{r} \partial_{t} \Delta^{\star} \phi+\left[\frac{1}{r^{2}} \Delta^{\star} \phi, \phi\right]-\left[\frac{1}{r^{2}} \Delta^{\star} \psi, \psi\right]=0, \quad(r, z) \in \mathcal{D} .
$$

Magnetic part: Using the rotational expression $\mathbf{B}=\nabla \wedge(\psi \nabla \theta)$, we get

$$
\int_{\mathcal{T}} \nabla \wedge\left[\partial_{t}(\psi \nabla \theta)-\mathbf{u} \wedge \mathbf{B}\right] \cdot \nabla \wedge(\widetilde{\psi} \nabla \theta) d v=0, \quad \forall \widetilde{\psi} \in X_{0} \cap X_{\perp} .
$$

Integrating by parts, it yields

$$
\int_{\mathcal{T}}\left[\partial_{t}(\psi \nabla \theta)-\mathbf{u} \wedge \mathbf{B}\right] \cdot \nabla \theta \Delta \widetilde{\psi} d v=0
$$

where we used the fact that $\nabla \cdot(\widetilde{\psi} \nabla \theta)=0$ to simplify $\nabla \wedge(\nabla \wedge(\widetilde{\psi} \nabla \theta))=-\Delta \widetilde{\psi} \nabla \theta$. 
From the relations $\mathbf{u}=\nabla \phi \wedge \nabla \theta=\left(-\frac{1}{r} \partial_{z} \phi, 0, \frac{1}{r} \partial_{r} \phi\right)$ and $\mathbf{B}=\nabla \psi \wedge \nabla \theta=\left(-\frac{1}{r} \partial_{z} \psi, 0, \frac{1}{r} \partial_{r} \psi\right)$, we deduce

$$
(\mathbf{u} \wedge \mathbf{B}) \cdot e_{\theta}=-\frac{1}{r^{2}} \partial_{r} \phi \partial_{z} \psi+\frac{1}{r^{2}} \partial_{r} \psi \partial_{z} \phi=\frac{1}{r^{2}}[\psi, \phi]
$$

and then, the magnetic equation (36) with (37) writes

$$
\int_{\mathcal{T}}\left(\partial_{t} \psi-\frac{1}{r}[\psi, \phi]\right) \Delta \widetilde{\psi} d v=0
$$

We observe that $\partial_{t} \psi-\frac{1}{r}[\psi, \phi] \in X_{\perp}$ and that $\widetilde{\psi} \in X_{\perp} \cap X_{0}$. So we finally get

$$
\partial_{t} \psi-\frac{1}{r}[\psi, \phi]=Q, \quad \Delta Q=0 .
$$

Homogeneous boundary conditions for $\psi$ and $\phi$ yield that $\partial_{t} \psi=[\psi, \phi]=0$ on the boundary: we conclude that $Q=0$ and

$$
\partial_{t} \psi-\frac{1}{r}[\psi, \phi]=0, \quad(x, z) \in \mathcal{D}
$$

Energy check: At the abstract level, it can be check either starting from (18). To verified that the boundary terms are zeroed, one must use the boundary conditions. This is performed by multiplying the first equation of (30) by $\frac{1}{r} \Delta^{*} \psi$, the second equation of (30) by $\frac{1}{r} \phi$, and by series of integration by parts which use all boundary conditions.

The proof is ended.

3.4.2. A second poloidal model Let $\rho_{0}>0$ be the initial density which is constant in the toroidal direction but not necessarily constant in the poloidal plane, that is $\rho_{0}=\rho_{0}(r, z)$. We will see that the next reduced model preserves the initial density $\rho=\rho_{0}$ : the model is incompressible (in time). On the other hand the entropy is assumed uniformly constant in space $\left(s=s_{0}=1\right.$ for example).

The set of constraints that we consider is

$$
\mathcal{K}_{2}=\operatorname{Span}_{(a, \phi, \psi, b) \in X \times X_{\perp}^{2} \times X}\left(a, \frac{1}{\rho_{0}} \nabla \phi \wedge \nabla \theta, \nabla \psi \wedge \nabla \theta, 0\right) \subset X^{8} .
$$

Proposition 13 Assume Dirichlet-Neumann boundary data for the magnetic and velocity potentials, $\psi=\phi=$ $\partial_{n} \phi=0$ on $\partial \Omega$, and assume $s_{0} \equiv 1$. The isentropic reduced model deduced from $\mathcal{K}_{2}$ is incompressible $\rho=\rho_{0}$ and writes in strong form

$$
\left\{\begin{array}{l}
\partial_{t} \psi=\frac{1}{\rho r}[\psi, \phi] \\
\partial_{t} \omega=\rho r\left[\frac{1}{(\rho r)^{2}} \omega, \phi\right]-\rho r\left[\frac{1}{\rho r^{2}} \Delta^{\star} \psi, \psi\right]
\end{array}\right.
$$

where the vorticity is $\omega=\Delta_{\rho} \phi$ and the operator $\Delta_{\rho}$ is defined by $\Delta_{\rho} g=\Delta^{\star} g-\frac{1}{\rho} \nabla \rho \cdot \nabla g=\rho r \nabla \cdot\left(\frac{1}{\rho r} \nabla g\right)$.

This model is endowed with the formal energy identity

$$
\frac{d}{d t} \int_{\mathcal{D}} \frac{|\nabla \psi|^{2}}{2 r}+\frac{|\nabla \phi|^{2}}{2 \rho r} d r d z=0 .
$$

Remark 14 This model has been derived in [15] by a completely different method, very similar to the usual one in plasma physics literature.

Proof. Starting with (17) related to the MHD model (22), with test functions $Z=\left(\widetilde{a}, \frac{1}{\rho_{0}} \nabla \widetilde{\phi} \wedge \nabla \theta, \nabla \widetilde{\psi} \wedge \nabla \theta, 0\right) \in$ $d \mathcal{K}$, one writes

$$
\left\{\begin{array}{l}
\int_{\mathcal{T}}\left(\partial_{t} \rho+\nabla \cdot(\rho \mathbf{u})\right) \widetilde{a} d v=0 \\
\int_{\mathcal{T}}\left[\partial_{t}(\rho \mathbf{u})+\nabla \cdot(\rho \mathbf{u} \otimes \mathbf{u})+\nabla p-(\nabla \wedge \mathbf{B}) \wedge \mathbf{B}\right] \cdot \frac{1}{\rho_{0}} \nabla \widetilde{\phi} \wedge \nabla \theta d v=0 \\
\int_{\mathcal{T}}\left[\partial_{t} \mathbf{B}-\nabla \wedge(\mathbf{u} \wedge \mathbf{B})\right] \cdot \nabla \widetilde{\psi} \wedge \nabla \theta d v=0, \\
\mathbf{u}=\frac{1}{\rho_{0}} \nabla \phi \wedge \nabla \theta, \quad \mathbf{B}=\nabla \psi \wedge \nabla \theta .
\end{array}\right.
$$


Thermodynamic part: Since this equality is satisfied for all $\widetilde{a} \in \mathbb{R}$, the mass conservation is satisfied in the whole domain. Moreover: $\mathbf{u}=\frac{1}{\rho_{0}} \nabla \phi \wedge \nabla \theta \Longrightarrow \rho_{0} \mathbf{u}=\nabla \wedge(\phi \nabla \theta) \Longrightarrow \nabla \cdot\left(\rho_{0} \mathbf{u}\right)=\nabla \cdot \nabla \wedge(\phi \nabla \theta)=0 \Longrightarrow \partial_{t} \rho_{0}=0$. Then, the density $\rho$ is conserved for all time : $\forall t>0, \rho(t)=\rho_{0}$. The constraint on the entropy is trivial.

Velocity part: Recalling that $\nabla \widetilde{\phi} \wedge \nabla \theta=\nabla \wedge(\widetilde{\phi} \nabla \theta)$, and integrating by parts, we get

$$
\int_{\mathcal{T}} \nabla \wedge\left[\partial_{t} \mathbf{u}+\mathbf{u} \cdot \nabla \mathbf{u}+\frac{\nabla p}{\rho}-\frac{(\nabla \wedge \mathbf{B}) \wedge \mathbf{B}}{\rho}\right] \cdot \nabla \theta \widetilde{\phi} d v=0
$$

Due to the isentropic condition the pressure can be expressed as a function of the density only, that is $p=p(\rho)$ : so the quantity $\frac{\nabla p}{\rho}$ is a gradient and its rotational vanishes. The preceding integral equation is satisfied for all arbitrary test function $\widetilde{\phi} \in X_{\perp} \cap X_{0}$. Moreover the other term also belongs to $X_{\perp}$. So

$$
\nabla \wedge\left[\partial_{t} \mathbf{u}+\mathbf{u} \cdot \nabla \mathbf{u}-\frac{(\nabla \wedge \mathbf{B}) \wedge \mathbf{B}}{\rho}\right] \cdot e_{\theta}=0, \quad \mathbf{x} \in \mathcal{T} .
$$

First of all, we write $\mathbf{u}=\frac{1}{\rho} \nabla \phi \wedge \nabla \theta=\left(-\frac{1}{\rho r} \partial_{z} \phi, 0, \frac{1}{\rho r} \partial_{r} \phi\right)$, and note $\alpha_{\rho}=-\frac{1}{\rho r} \partial_{z} \phi$ and $\beta_{\rho}=\frac{1}{\rho r} \partial_{r} \phi$ so that $\mathbf{u}=\left(\alpha_{\rho}, 0, \beta_{\rho}\right)$. Then, we calculate

$$
\begin{aligned}
(\nabla \wedge \mathbf{u}) \cdot e_{\theta} & =\partial_{z}\left(-\frac{1}{\rho r} \partial_{z} \phi\right)-\partial_{r}\left(\frac{1}{\rho r} \partial_{r} \phi\right) \\
& =-\frac{1}{\rho r} \partial_{z}^{2} \phi-\frac{1}{\rho r} \partial_{r}^{2} \phi+\frac{1}{\rho r^{2}} \partial_{r} \phi+\frac{1}{\rho^{2} r}\left(\partial_{r} \rho \partial_{r} \phi+\partial_{z} \rho \partial_{z} \phi\right) \\
& =-\frac{1}{\rho r}\left(\Delta_{\perp} \phi-\frac{1}{r} \partial_{r} \phi-\frac{1}{\rho}(\nabla \rho \cdot \nabla \phi)\right) \\
& =-\frac{1}{\rho r}\left(\Delta^{\star} \phi-\frac{1}{\rho}(\nabla \rho \cdot \nabla \phi)\right)
\end{aligned}
$$

Then, noting $\Delta_{\rho} \phi=\Delta^{\star} \phi-\frac{1}{\rho} \nabla \rho \cdot \nabla \phi$, we get :

$$
\nabla \wedge\left(\partial_{t} \mathbf{u}\right) \cdot e_{\theta}=-\frac{1}{\rho r} \partial_{t} \Delta_{\rho} \phi
$$

Next, $\mathbf{u} \cdot \nabla \mathbf{u}=\left(\alpha_{\rho} \partial_{r} \alpha_{\rho}+\beta_{\rho} \partial_{z} \alpha_{\rho}, 0, \alpha_{\rho} \partial_{r} \beta_{\rho}+\beta_{\rho} \partial_{z} \beta_{\rho}\right)^{t}$ and

$$
\begin{array}{r}
\nabla \wedge(\mathbf{u} \cdot \nabla \mathbf{u}) \cdot e_{\theta}=\partial_{z}\left(\alpha_{\rho} \partial_{r} \alpha_{\rho}+\beta_{\rho} \partial_{z} \alpha_{\rho}\right)-\partial_{r}\left(\alpha_{\rho} \partial_{r} \beta_{\rho}+\beta_{\rho} \partial_{z} \beta_{\rho}\right) \\
=\alpha_{\rho} \partial_{r}\left(\partial_{z} \alpha_{\rho}-\partial_{r} \beta_{\rho}\right)+\beta_{\rho} \partial_{z}\left(\partial_{z} \alpha_{\rho}-\partial_{r} \beta_{\rho}\right)+\left(\partial_{z} \alpha_{\rho}-\partial_{r} \beta_{\rho}\right)\left(\partial_{r} \alpha_{\rho}+\partial_{z} \beta_{\rho}\right) .
\end{array}
$$

Since $\partial_{z} \alpha_{\rho}-\partial_{r} \beta_{\rho}=\partial_{z}\left(-\frac{1}{\rho r} \partial_{z} \phi\right)-\partial_{r}\left(\frac{1}{\rho r} \partial_{r} \phi\right)=-\frac{1}{\rho r} \Delta_{\rho} \phi$ and

$$
\begin{aligned}
\partial_{r} \alpha_{\rho}+\partial_{z} \beta_{\rho} & =\partial_{r}\left(-\frac{1}{\rho r} \partial_{z} \phi\right)+\partial_{z}\left(\frac{1}{\rho r} \partial_{r} \phi\right) \\
& =-\frac{1}{\rho r} \partial_{r} \partial_{z} \phi+\frac{1}{\rho r^{2}} \partial_{z} \phi+\frac{1}{\rho^{2} r} \partial_{r} \rho \partial_{z} \phi+\frac{1}{\rho r} \partial_{z} \partial_{r} \phi-\frac{1}{\rho^{2} r} \partial_{z} \rho \partial_{r} \phi \\
& =-\partial_{r}\left(\frac{1}{\rho r}\right) \partial_{z} \phi+\partial_{z}\left(\frac{1}{\rho r}\right) \partial_{r} \phi=-\left[\frac{1}{\rho r}, \phi\right]
\end{aligned}
$$

we finally obtain

$$
\begin{aligned}
& \nabla \wedge(\mathbf{u} \cdot \nabla \mathbf{u}) \cdot e_{\theta} \\
= & -\frac{1}{\rho r} \partial_{z} \phi \partial_{r}\left(-\frac{1}{\rho r} \Delta_{\rho} \phi\right)+\frac{1}{\rho r} \partial_{r} \phi \partial_{z}\left(-\frac{1}{\rho r} \Delta_{\rho} \phi\right)+\frac{1}{\rho r} \Delta^{\star} \phi\left[\frac{1}{\rho r}, \phi\right] \\
= & \partial_{z} \phi \partial_{r}\left(\frac{1}{(\rho r)^{2}} \Delta_{\rho} \phi\right)-\partial_{r} \phi \partial_{z}\left(\frac{1}{(\rho r)^{2}} \Delta_{\rho} \phi\right)\left[\frac{1}{(\rho r)^{2}} \Delta_{\rho} \phi, \phi\right]
\end{aligned}
$$


Next, we deal with the magnetic term. From $\mathbf{B}=\nabla \psi \wedge \nabla \theta=\left(-\frac{1}{r} \partial_{z} \psi, 0, \frac{1}{r} \partial_{r} \psi\right)$ and $\nabla \wedge \mathbf{B}=\left(0, \partial_{z}\left(-\frac{1}{r} \partial_{z} \psi\right)-\partial_{r}\left(\frac{1}{r} \partial_{r} \psi\right)\right.$, $\left(0,-\frac{\Delta^{\star} \psi}{r}, 0\right)$, one obtains $\frac{(\nabla \wedge \mathbf{B}) \wedge \mathbf{B}}{\rho}=\left(-\frac{1}{\rho r} \partial_{r} \psi \frac{\Delta^{\star} \psi}{r}, 0,-\frac{1}{\rho r} \partial_{z} \psi \frac{\Delta^{\star} \psi}{r}\right)$ and

$$
\begin{aligned}
\nabla \wedge\left(\frac{(\nabla \wedge \mathbf{B}) \wedge \mathbf{B}}{\rho}\right) \cdot e_{\theta} & =\partial_{z}\left(-\frac{1}{\rho r} \partial_{r} \psi \frac{\Delta^{\star} \psi}{r}\right)+\partial_{r}\left(\frac{1}{\rho r} \partial_{z} \psi \frac{\Delta^{\star} \psi}{r}\right) \\
& =-\partial_{r} \psi \partial_{z}\left(\frac{1}{\rho r^{2}} \Delta^{\star} \psi\right)+\partial_{z} \psi \partial_{r}\left(\frac{1}{\rho r^{2}} \Delta^{\star} \psi\right) \\
& =\left[\frac{1}{\rho r^{2}} \Delta^{\star} \psi, \psi\right]
\end{aligned}
$$

Introducing (42), (43) and (44) in the equation (41), it comes

$$
-\frac{1}{\rho r} \partial_{t} \Delta_{\rho} \phi+\left[\frac{1}{\rho r^{2}} \Delta_{\rho} \phi, \phi\right]-\left[\frac{1}{\rho r^{2}} \Delta^{\star} \psi, \psi\right]=0, \quad(x, z) \in \mathcal{D} .
$$

Magnetic part: Using the rotational expression $\mathbf{B}=\nabla \wedge(\psi \nabla \theta)$, we get

$$
\int_{\mathcal{T}} \nabla \wedge\left[\partial_{t}(\psi \nabla \theta)-\mathbf{u} \wedge \mathbf{B}\right] \cdot \nabla \wedge(\widetilde{\psi} \nabla \theta) d v=0
$$

and, integrating by parts and by $\nabla \theta=\frac{e_{\theta}}{r}$, it yields for $\widetilde{\psi} \in X_{\perp} \cap X_{0}$

$$
\int_{\mathcal{T}}\left[\partial_{t}\left(\frac{\psi e_{\theta}}{r}\right)-\mathbf{u} \wedge \mathbf{B}\right] \cdot \frac{e_{\theta}}{r} \Delta \widetilde{\psi} d v=0 .
$$

From the relations $\mathbf{u}=\frac{1}{\rho} \nabla \phi \wedge \nabla \theta=\left(-\frac{1}{\rho r} \partial_{z} \phi, 0, \frac{1}{\rho r} \partial_{r} \phi\right)$ and $\mathbf{B}=\nabla \psi \wedge \nabla \theta=\left(-\frac{1}{r} \partial_{z} \psi, 0, \frac{1}{r} \partial_{r} \psi\right)$ we deduce

$$
(\mathbf{u} \wedge \mathbf{B}) \cdot e_{\theta}=-\frac{1}{\rho r^{2}} \partial_{r} \phi \partial_{z} \psi+\frac{1}{\rho r^{2}} \partial_{r} \psi \partial_{z} \phi=\frac{1}{\rho r^{2}}[\psi, \phi]
$$

and then, the magnetic equation writes

$$
\int_{\mathcal{T}} \frac{1}{r}\left(\partial_{t} \psi-\frac{1}{\rho r}[\psi, \phi]\right) \Delta \widetilde{\psi} d v=0
$$

As before, this weak equation being true for all $\widetilde{\psi} \in X_{\perp} \cap X_{0}$, it means that

$$
\frac{1}{r}\left(\partial_{t} \psi-\frac{1}{\rho r}[\psi, \phi]\right)=Q, \quad \Delta Q=0 .
$$

By hypothesis, homogeneous boundary conditions are required $\psi=\phi=0$ on the boundary. We conclude that $Q=0$ and

$$
\partial_{t} \psi-\frac{1}{\rho r}[\psi, \phi]=0, \quad(x, z) \in \mathcal{D} .
$$

Energy check: it can be verified by two methods, as in the proof of the proposition. Except that the second equation must be multiplied by $\frac{1}{\rho r} \phi$, which is algebraically compatible with $\omega=\rho r \nabla \cdot\left(\frac{1}{\rho r} \nabla \varphi\right)$. Detailed verifications are in [15]. The proof is ended.

3.4.3. A third poloidal model As before the initial density $\rho_{0}(r, z)$ is constant in the toroidal direction but not necessarily constant in the poloidal cut. We introduce a non constant "forcing term" $F=F(r, z)$ in the set of constraints, that is

$$
\mathcal{K}_{3}=V_{0}+\operatorname{Span}_{(a, \phi, \psi, b) \in X \times X_{\perp}^{2} \times X}\left(a, \frac{1}{\rho}_{0} \nabla \phi \wedge \nabla \theta, \nabla \psi \wedge \nabla \theta, b\right)
$$

with $V_{0}=(0,0, F \nabla \theta, 0)$.

With this choice the representation of the magnetic field is exactly $\mathbf{B}=F \nabla \theta+\nabla \psi \wedge \nabla \theta$. A non zero $F$ is the minimum to represents correctly the helical structure of the magnetic field in Tokamaks [18]. 
Proposition 15 Assume Dirichlet-Neumann boundary data for the magnetic and velocity potentials: $\psi=\phi=$ $\partial_{n} \phi=0$ on $\partial \Omega$. The reduced model of (22) related to the adjoint variable set $\mathcal{K}_{3}$ is incompressible $\rho=\rho_{0}$ and writes

$$
\left\{\begin{array}{l}
\partial_{t} \psi=\frac{1}{\rho r}[\psi, \phi], \\
\partial_{t} \omega=\rho r\left[\frac{1}{(\rho r)^{2}} \omega, \phi\right]-\rho r\left[\frac{F}{\rho r^{2}}, F\right]-\rho r\left[\frac{1}{\rho r^{2}} \Delta^{\star} \psi, \psi\right]
\end{array}\right.
$$

where the vorticity is $\omega=\Delta_{\rho} \phi$.

The energy identity is

$$
\frac{d}{d t} \int_{\mathcal{D}} \frac{|\nabla \psi|^{2}}{2 r}+\frac{|\nabla \phi|^{2}}{2 \rho r} d r d z=\int_{\mathcal{D}}\left[\frac{F}{\rho r^{2}}, F\right] \phi d r d z
$$

The new term with respect to (39) is the source term $\left[\frac{F}{\rho r^{2}}, F\right]$. We will show in proposition 18 that it is essential to obtain the compatibility with the Grad-Shrafanov equation $[3,41]$ for equilibrium in Tokamaks. If $F=F_{0}$ is constant in space, the source term vanishes: it also vanishes if $\rho r^{2}$ is a constant.

Remark 16 An interpretation of this energy balance is as follows. First the physical energy is the sum of the internal energy, the kinetic energy and the magnetic energy $\rho e=\rho \varepsilon+\frac{\rho|\mathbf{u}|^{2}}{2}+\frac{|\mathbf{B}|^{2}}{2}$. Plugging the chosen representation $\mathcal{K}_{3}$, we obtain

$$
\rho e=\left(\rho \varepsilon+\frac{\left|F_{0} \nabla \theta\right|^{2}}{2}\right)+\frac{\left|\nabla \phi \wedge \mathbf{e}_{\theta}\right|^{2}}{2 \rho r^{2}}+\frac{\left|\nabla \psi \wedge \mathbf{e}_{\theta}\right|^{2}}{2 r^{2}}=K+\frac{|\nabla \phi|^{2}}{2 \rho r^{2}}+\frac{|\nabla \psi|^{2}}{2 r^{2}}
$$

since $\psi, \phi \in X_{\perp}$. The term $K$ is independent of the time variable and vanishes after derivation with respect to $t$. The time derivative of the integral (with weight rdrdz) of the remaining term is exactly the left hand side of the energy balance (48). Notice that the linear part of (18) vanishes

$$
\frac{d}{d t} \int_{\Omega} V_{0}(\mathbf{x}) \cdot U=\frac{d}{d t} \int_{\Omega}(F \nabla \theta) \cdot((F \nabla \theta+\nabla \psi \wedge \theta) d x d y d z=0 .
$$

Since the model is hyperbolic compatible, the right hand side of (48) is by construction equal to the right hand side of (18). This non trivial fact deserves a separate verification. Indeed one has the series of equalities (discarding all terms on the boundary)

$$
\begin{aligned}
& \int_{\Omega} \nabla V_{0}(\mathbf{x}): f(U) d x d y d z=-\int_{\Omega} V_{0}(\mathbf{x}) \cdot \nabla \cdot f(U) d x d y d z \\
= & \int_{\Omega} F \nabla \theta \cdot \nabla \wedge(\mathbf{u} \wedge \mathbf{B}) d x d y d z=\int_{\Omega} \nabla \wedge(F \nabla \theta) \cdot \mathbf{u} \wedge \mathbf{B} d x d y d z \\
= & \int_{\Omega}(\nabla F \wedge \nabla \theta) \cdot\left(\left(\frac{1}{\rho} \nabla \phi \wedge \nabla \theta\right) \wedge(F \nabla \theta+\nabla \psi \wedge \nabla \theta)\right) d x d y d z .
\end{aligned}
$$

Simplifications due to $\nabla \psi \cdot \mathbf{e}_{\theta}=\nabla \phi \cdot \mathbf{e}_{\theta}=\nabla F \cdot \mathbf{e}_{\theta}=0$ yield

$$
\int_{\Omega} \nabla V_{0}(\mathbf{x}): f(U) d x d y d z=-\int_{\Omega} \frac{F}{\rho r^{3}}\left(\nabla F \wedge \mathbf{e}_{\theta}\right) \cdot \nabla \phi d x d y d z=-2 \pi \int_{\mathcal{D}} \frac{F}{\rho r^{2}}[\phi, F] d r d z .
$$

One more integration by parts of the Poisson bracket shows that

$$
\int_{\Omega} \nabla V_{0}(\mathbf{x}): f(U) d x d y d z=2 \pi \int_{\mathcal{D}}\left[\frac{F}{\rho r^{2}}, F\right] \phi d r d z
$$

which is exactly the right hand side of (48), up to the factor $2 \pi$ of course.

Proof. Since this model is very close to the previous one, we concentrate only on the important part of the proof. Starting with (17) related to the MHD model (22), with test functions $Z=\left(0, \frac{1}{\rho_{0}} \nabla \widetilde{\phi} \wedge \nabla \theta, \nabla \widetilde{\psi} \wedge \nabla \theta, 0\right) \in d \mathcal{K}$, one writes

$$
\left\{\begin{array}{l}
\int_{\mathcal{T}}\left[\partial_{t}(\rho \mathbf{u})+\nabla \cdot(\rho \mathbf{u} \otimes \mathbf{u})+\nabla p-(\nabla \wedge \mathbf{B}) \wedge \mathbf{B}\right] \cdot \frac{1}{\rho_{0}} \nabla \widetilde{\phi} \wedge \nabla \theta=0 \\
\int_{\mathcal{T}}\left[\partial_{t} \mathbf{B}-\nabla \wedge(\mathbf{u} \wedge \mathbf{B})\right] \cdot \nabla \widetilde{\psi} \wedge \nabla \theta=0 \\
\mathbf{u}=\frac{1}{\rho_{0}} \nabla \phi \wedge \nabla \theta, \quad \mathbf{B}=F \nabla \theta+\nabla \psi \wedge \nabla \theta
\end{array}\right.
$$

The hydrodynamic $(\rho, s)$ part can be treated as for the second poloidal model. 
Velocity part: The only contribution of the additional term $F \nabla \theta$ in $\mathbf{B}$ appears in the Lorentz term of the velocity equation:

$$
\int_{\mathcal{T}}(\nabla \wedge \mathbf{B}) \wedge \mathbf{B} \cdot \frac{1}{\rho} \nabla \widetilde{\phi} \wedge \nabla \theta=\int_{\mathcal{T}} \nabla \wedge\left(\frac{(\nabla \wedge \mathbf{B}) \wedge \mathbf{B}}{\rho}\right) \cdot \Delta \widetilde{\phi} \nabla \theta
$$

The expression of $\mathbf{B}$ gives $\mathbf{B}=F \nabla \theta+\nabla \psi \wedge \nabla \theta=\left(-\frac{1}{r} \partial_{z} \psi, \frac{F}{r}, \frac{1}{r} \partial_{r} \psi\right)^{t}$,

$$
\begin{aligned}
\nabla \wedge \mathbf{B} & =\left(-\frac{1}{r} \partial_{z} F, \partial_{z}\left(-\frac{1}{r} \partial_{z} \psi\right)-\partial_{r}\left(\frac{1}{r} \partial_{r} \psi\right), \frac{1}{r} \partial_{r} F\right)^{t} \\
& =\left(-\frac{1}{r} \partial_{z} F,-\frac{\Delta^{\star} \psi}{r}, \frac{1}{r} \partial_{r} F\right)^{t}, \\
& \frac{(\nabla \wedge \mathbf{B}) \wedge \mathbf{B}}{\rho}=\left(\begin{array}{c}
-\frac{F}{\rho r^{2}} \partial_{r} F-\frac{1}{\rho r} \partial_{r} \psi \frac{\Delta^{\star} \psi}{r}, \\
-\frac{1}{\rho r^{2}} \partial_{r} \psi \partial_{z} F+\frac{1}{\rho r^{2}} \partial_{z} \psi \partial_{r} F \\
-\frac{F}{\rho r^{2}} \partial_{z} F-\frac{1}{\rho r} \partial_{z} \psi \frac{\Delta^{\star} \psi}{r}
\end{array}\right)
\end{aligned}
$$

and finally

$$
\begin{aligned}
& \nabla \wedge\left(\frac{(\nabla \wedge \mathbf{B}) \wedge \mathbf{B}}{\rho}\right) \cdot e_{\theta} \\
= & -\partial_{z}\left(\frac{F}{\rho r^{2}} \partial_{r} F+\frac{1}{\rho r} \partial_{r} \psi \frac{\Delta^{\star} \psi}{r}\right)+\partial_{r}\left(\frac{F}{\rho r^{2}} \partial_{z} F+\frac{1}{\rho r} \partial_{z} \psi \frac{\Delta^{\star} \psi}{r}\right) \\
= & {\left[\frac{F}{\rho r^{2}}, F\right]+\left[\frac{1}{\rho r^{2}} \Delta^{\star} \psi, \psi\right] . }
\end{aligned}
$$

This is the reason of the quadratic source term in the vorticity equation.

Magnetic part: The additional part $F \nabla \theta$ in $\mathbf{B}$ has no contribution to the magnetic equation. Indeed,

$$
\begin{gathered}
\int_{\mathcal{T}}\left[\partial_{t} F \nabla \theta-\nabla \wedge(\mathbf{u} \wedge F \nabla \theta)\right] \cdot \nabla \widetilde{\psi} \wedge \nabla \theta \\
=\int_{\mathcal{T}} \partial_{t} F \nabla \theta \cdot(\nabla \widetilde{\psi} \wedge \nabla \theta)-(\mathbf{u} \wedge F \nabla \theta) \cdot \Delta \widetilde{\psi} \nabla \theta=0
\end{gathered}
$$

since $\partial_{t} F=0$ and $(\mathbf{u} \wedge \nabla \theta) \cdot \nabla \theta=0$.

Energy balance: it can be checked as for the previous models.

\subsection{Toroidal dependency}

We now consider a more complete case with toroidal dependency which structure is close to what is usually encountered in plasma physics articles $[6,10,11,23,26,40,42,46,50]$. The result is increasing algebraic complexity even if we do not introduce parallel velocity for the sake of simplicity. Parallel velocity is used in almost all quoted physical references and also in the preprint [45].

The starting point is the set

$$
\mathcal{K}_{4}=V_{0}+\operatorname{Span}_{(a, b) \in \mathbb{R}^{2},(\phi, \psi) \in X^{2}}\left(a, \frac{1}{\rho_{0}} \nabla \phi \wedge \nabla \theta, \nabla \psi \wedge \nabla \theta, b\right)
$$

with a forcing term $V_{0}=(0,0, F \nabla \theta, 0)$ and $F=F(r, z)$. A feature of this model is that all functions $\psi$ and $\phi$ have dependency with respect to the toroidal variable $\theta$. As before the magnetic forcing $F$ does not depend on the toroidal variable: $\nabla F \perp \nabla \theta$. The velocity is perpendicular $\mathbf{u}=\mathbf{u}_{\perp}=G \nabla \phi \wedge \nabla \theta$ with $G=\frac{1}{\rho_{0}}$. Similar formulas with parallel velocity and an arbitrary coefficient independent of the toroidal variable $\nabla G^{\rho_{0}} \perp \nabla \theta$ are to be found in [45]. We will assume for the simplicity of notations that $F=F_{0}$ is constant.

Proposition 17 Assume homogeneous boundary conditions $\psi=\phi=0$. Assume $\rho(t=0)=\rho_{0}$ and $F=F_{0}$ is constant. The reduced model based on $\mathcal{K}_{4}$ is incompressible $\rho=\rho_{0}$ and can be written under the strong form

$$
\left\{\begin{array}{lr}
\partial_{t} \psi=\frac{1}{\rho r}[\psi, \phi]+\frac{F_{0}}{\rho r^{2}} \partial_{\theta} \phi+Q, & \mathbf{x} \in \mathcal{T}, \\
\partial_{t} \omega=\rho r\left[\frac{1}{(\rho r)^{2}} \omega, \phi\right]-\rho r\left[\frac{1}{\rho r^{2}} \Delta^{\star} \psi, \psi\right]+\frac{1}{r^{2}} F_{0} \Delta^{\star \star \star} \partial_{\theta} \psi, \mathbf{x} \in \mathcal{T}, \\
\omega=\Delta^{\star} \phi, \\
\mathbf{x} \in \mathcal{T} .
\end{array}\right.
$$


The new diffusion operator is $\Delta^{\star \star \star} f=\rho r^{3}\left(\partial_{r}\left(\frac{1}{\rho r^{3}} \partial_{r} f\right)+\partial_{z}\left(\frac{1}{\rho r^{3}} \partial_{z} f\right)\right)$. The source term $Q$ is solution for all $\theta$ of a series of poloidal variational formulations, $a(Q(\theta), \widetilde{\psi})=\left(g_{\theta}, \widetilde{\psi}\right)$ for all $\widetilde{\psi} \in V_{\perp}$, where $V_{\perp} \subset H^{1}(\mathcal{D})$ encounters of the boundary condition, typically $V_{\perp}=H_{0}^{1}(\mathcal{D})$, and $\widetilde{\psi}$ denotes any test function in $V_{\perp}$. The bilinear form is

$$
a(Q, \widetilde{\psi})=\int_{\mathcal{D}} \frac{1}{r}\left(\partial_{r} Q \partial_{r} \widetilde{\psi}+\partial_{z} Q \partial_{z} \widetilde{\psi}\right)
$$

The right hand side is

$$
\left(g_{\theta}, \widetilde{\psi}\right)=\int \frac{1}{r^{3}}\left(\partial_{\theta}\left(-\phi \partial_{z}\left(\frac{F_{0}}{\rho}\right) \partial_{z} \widetilde{\psi}+\partial_{\theta}\left(-\phi \partial_{r} \frac{F_{0}}{\rho}\right) \partial_{r} \widetilde{\psi}\right)+2 \int \frac{F_{0}}{\rho r^{4}} \partial_{\theta} \phi \partial_{r} \widetilde{\psi}\right.
$$

The energy balance is

$$
\frac{1}{2} \frac{d}{d t} \int_{\Omega} \frac{1}{r}\left(\left|\nabla_{r, z} \psi\right|^{2}+\left|\nabla_{r, z} \phi\right|^{2}\right)=0
$$

The equation of $Q$ is also conveniently written in weak or variational form. The strong form is

$$
\begin{gathered}
\Delta^{\star} Q=r \partial_{z}\left(\frac{1}{r^{3}} \partial_{\theta}\left(-\phi \partial_{z}(F G)+\psi \partial_{z} \lambda\right)\right) \\
+r \partial_{r}\left(\frac{1}{r^{3}} \partial_{\theta}\left(-\phi \partial_{r}(F G)+\psi \partial_{r} \lambda\right)+\frac{2}{r^{4}} \partial_{\theta}(F G \phi-\lambda \psi)\right) .
\end{gathered}
$$

Proof. Using the same method as before, the thermodynamic equations disappear. The details of the proof are given for a general $F$. Testing against all possible test functions $\widetilde{\phi}$ one obtains after integration by parts

$$
\nabla \theta \cdot\left(\nabla \wedge \frac{1}{\rho}\left[\partial_{t}(\rho \mathbf{u})+\nabla \cdot(\rho \mathbf{u} \otimes \mathbf{u})+\nabla p-(\nabla \wedge \mathbf{B}) \wedge \mathbf{B}\right]\right)=0 .
$$

We notice that it can be simplified under the form

$$
\nabla \theta \cdot\left(\nabla \wedge\left[\partial_{t} \mathbf{u}+\mathbf{u} \cdot \nabla \mathbf{u}-\frac{1}{\rho}(\nabla \wedge \mathbf{B}) \wedge \mathbf{B}\right]\right)=0
$$

Once again the equation is independent of the pressure $p$. The first part of the equation explicitly depends on $\mathbf{u}$ for which a length but elementary verification shows that (42) and (43) are not changed. That is this part of the model does not contain derivatives with respect to $\theta$ and so is identical to (39). It remains to determine the scalar product against $\mathbf{e}_{\theta}$ of the magnetic part of this equation. One still has $\mathbf{B}=\frac{1}{r}\left(-\partial_{z} \psi, F, \partial_{r} \psi\right)^{t}$. An additional term show up in the current

$$
\nabla \wedge \mathbf{B}=\left(-\frac{1}{r} \partial_{z} F,-\frac{\Delta^{\star} \psi}{r}, \frac{1}{r} \partial_{r} F\right)^{t}+\frac{1}{r^{2}}\left(\partial_{r \theta}^{2} \psi, 0, \partial_{z \theta}^{2} \psi\right)^{t}
$$

Careful calculations show that

$$
\begin{gathered}
\mathbf{e}_{\theta} \cdot \nabla \wedge\left[\frac{1}{\rho}(\nabla \wedge \mathbf{B}) \wedge \mathbf{B}\right]=\left[\frac{1}{\rho r^{2}} \Delta^{\star} \psi, \psi\right] \\
-\partial_{r}\left(\frac{F}{\rho r^{3}} \partial_{r \theta}^{2} \psi\right)-\partial_{z}\left(\frac{F}{\rho r^{3}} \partial_{z \theta}^{2} \psi\right)
\end{gathered}
$$

from which one recovers the vorticity part of the system (52) after simplification $F=F_{0}$.

The magnetic equation needs more attention. The weak form writes

$$
\int_{\mathcal{T}}\left(\partial_{t} \mathbf{B}-\nabla \wedge(\mathbf{u} \wedge \mathbf{B})\right) \cdot \nabla \wedge(\widetilde{\psi} \nabla \theta)=0 .
$$

Since by hypothesis $\partial_{t} \mathbf{B}=\nabla \wedge(\psi \nabla \theta)$, one can substitute and integrate by parts to obtain

$$
\int\left(\partial_{t} \psi \nabla \theta-\mathbf{u} \wedge \mathbf{B}\right) \cdot \nabla \wedge \nabla \wedge(\widetilde{\psi} \nabla \theta) d x d y d z=0
$$


Let us denote $\widetilde{\mathbf{B}}=\nabla \wedge(\widetilde{\psi} \nabla \theta)$ and $\widetilde{\mathbf{J}}=\nabla \wedge \widetilde{\mathbf{B}}$. Careful calculations show that since $\widetilde{\mathbf{B}}=-\frac{\partial_{z} \widetilde{\psi}}{r} \mathbf{e}_{r}+\frac{\partial_{r} \widetilde{\psi}}{r} \mathbf{e}_{z}$ and $\nabla \wedge \mathbf{e}_{r}=\nabla \wedge \mathbf{e}_{z}=0$, then

One also has

$$
\widetilde{\mathbf{J}}=-\frac{1}{r} \Delta^{\star} \widetilde{\psi} \mathbf{e}_{\theta}+\frac{1}{r^{2}}\left(\partial_{r \theta}^{2} \widetilde{\psi} \mathbf{e}_{r}+\partial_{z \theta}^{2} \widetilde{\psi} \mathbf{e}_{z}\right)
$$

$$
\begin{aligned}
\mathbf{u} & \wedge \mathbf{B}=\left(\frac{1}{\rho} \nabla \phi \wedge \nabla \theta\right) \wedge(F \nabla \theta+\nabla \psi \wedge \nabla \theta) \\
& =\frac{1}{\rho r^{2}}[\psi, \phi] \mathbf{e}_{\theta}-\frac{F}{\rho r^{2}}\left(\partial_{z} \phi \mathbf{e}_{z}+\partial_{r} \phi \mathbf{e}_{r}\right) .
\end{aligned}
$$

The measure of integration is $d x d y d z=2 \pi r d r d \theta d z$. Substitutions in (53) yield

$$
\begin{gathered}
\int\left(\left(-\frac{1}{r} \partial_{t} \psi+\frac{1}{\rho r^{2}}[\psi, \phi]\right) \Delta^{\star} \widetilde{\psi}\right. \\
\left.+\frac{1}{\rho r^{3}}\left(F \partial_{z} \phi\right) \partial_{z \theta}^{2} \widetilde{\psi}+\frac{1}{\rho r^{4}}\left(F \partial_{r} \phi\right) \partial_{r \theta}^{2} \widetilde{\psi}\right) d r d \theta d z=0 .
\end{gathered}
$$

It is convenient to define what we call a correction term $Q$ as

$$
Q=\partial_{t} \psi-\frac{1}{\rho r}[\psi, \phi]-\frac{1}{r^{2}} \partial_{\theta}\left(\frac{F \phi}{\rho}\right)
$$

where we keep $\frac{F}{\rho}$ inside the $\partial_{\theta}$ to shorten the notations. We get

$$
\begin{gathered}
\int\left(-\frac{1}{r} Q-\frac{1}{r^{3}} \partial_{\theta}\left(\frac{F \phi}{\rho}\right)\right) \Delta^{\star} \widetilde{\psi} \\
+\int\left(\frac{1}{r^{3}}\left(\frac{F}{\rho} \partial_{z} \phi\right) \partial_{z \theta}^{2} \widetilde{\psi}+\frac{1}{r^{3}}\left(\frac{F}{\rho} \partial_{r} \phi\right) \partial_{r \theta}^{2} \widetilde{\psi}\right) d r d \theta d z=0 .
\end{gathered}
$$

Integration by parts with respect to the Grad-Shafranov operator and to the toroidal variable $\theta$ yields

$$
\begin{gathered}
\int \frac{1}{r}\left(\partial_{r} Q \partial_{r} \widetilde{\psi}+\partial_{z} Q \partial_{z} \widetilde{\psi}\right) d r d \theta d z=\int \frac{1}{r^{3}} \partial_{\theta}\left(\frac{F \phi}{\rho}\right) \Delta^{\star} \widetilde{\psi} \\
+\int\left(\frac{1}{r^{3}} \partial_{\theta}\left(\frac{F}{\rho} \partial_{z} \phi\right) \partial_{z} \widetilde{\psi}+\frac{1}{r^{3}} \partial_{\theta}\left(\frac{F}{\rho} \partial_{r} \phi\right) \partial_{r} \widetilde{\psi}\right) \\
=\int \frac{1}{r^{3}} \partial_{\theta}\left(\frac{F}{\rho} \phi\right) \Delta^{\star} \widetilde{\psi} \\
+\int\left(\frac{1}{r^{3}} \partial_{z \theta}^{2}\left(\frac{F}{\rho} \phi\right) \partial_{z} \widetilde{\psi}+\frac{1}{r^{3}} \partial_{r \theta}^{2}\left(\frac{F}{\rho} \phi\right) \partial_{r} \widetilde{\psi}\right) . \\
+\int\left(\frac{1}{r^{3}} \partial_{\theta}\left(-\phi \partial_{z}(F G)+\psi \partial_{z} \lambda\right) \partial_{z} \widetilde{\psi}+\frac{1}{r^{3}} \partial_{\theta}\left(-\phi \partial_{r}(F G)+\psi \partial_{r} \lambda\right) \partial_{r} \widetilde{\psi}\right) .
\end{gathered}
$$

An integration by parts yields

We obtain

$$
\begin{gathered}
\int \frac{1}{r^{3}} \partial_{\theta}(F G \phi-\lambda \psi) \Delta^{\star} \widetilde{\psi}=-\int \frac{1}{r} \nabla_{r, z}\left(\frac{1}{r^{2}} \partial_{\theta}\left(\frac{F \phi}{\rho}\right)\right) \cdot \nabla_{r, z} \widetilde{\psi} \\
=-\int \frac{1}{r^{3}} \nabla_{r, z} \partial_{\theta}\left(\frac{F \phi}{\rho}\right) \cdot \nabla_{r, z} \widetilde{\psi}+2 \int \frac{1}{r^{4}} \partial_{\theta}\left(\frac{F \phi}{\rho}\right) \partial_{r} \widetilde{\psi}
\end{gathered}
$$

$$
\begin{gathered}
\int \frac{1}{r}\left(\partial_{r} Q \partial_{r} \widetilde{\psi}+\partial_{z} Q \partial_{z} \widetilde{\psi}\right) d r d \theta d z \\
=\int\left(\frac{1}{r^{3}} \partial_{\theta}\left(-\phi \partial_{z} \frac{F}{\rho}\right) \partial_{z} \widetilde{\psi}+\frac{1}{r^{3}} \partial_{\theta}\left(-\phi \partial_{r} \frac{F}{\rho}\right) \partial_{r} \widetilde{\psi}\right) \\
+2 \int \frac{1}{r^{4}} \partial_{\theta}\left(\frac{F \phi}{\rho}\right) \partial_{r} \widetilde{\psi} .
\end{gathered}
$$

Under this form, the correction term $Q$ is a the solution of a series of Grad-Shafranov-like weak formulations for all $\theta$. The energy identity is a consequence of proposition 19. It ends the proof. 


\section{Existence of a weak solution}

In this section we study the existence of a solution by means of analogy with the Teman-Lions theory. This makes necessary to incorporate some diffusive operators in the previous models. We have retained diffusive operators with two properties: they are the closest to what is in the physical literature; it is possible to prove existence of a weak solution using the regularity/compactness of the additional viscous part. Indeed a key tool to prove the existence of weak solutions is the energy identity which provides a control of the regularity of the solution. In "real life" physical situations and numerical simulations we are aware of, such diffusive operators are always present, even if with quite small viscous or resistive constants.

We concentrate on two different models, the first one with a non constant forcing term $F$ in poloidal geometry, the second one with a constant forcing term $F=F_{0}$ but in full toroidal geometry.

It is also possible to study a more general case with a non constant $F$ in the full toroidal geomatry but at the price of heavier notations.

For these two reasons we incorporate hereafter viscous and resistive operators to the reduced models derived before, and concentrate on the mathematical properties of a weak solution.

\subsection{Poloidal model with non constant forcing term}

We assume a non constant forcing term $F=F(r, z)$, but independent of the time variable and the toroidal variable $\theta$. For the sake of mathematical simplicity we consider isotropic viscosity and resistivity. This model is only poloidal since independent of the angular variable $\theta$. It writes

$$
\left\{\begin{array}{l}
\partial_{t} \psi=\frac{1}{\rho r}[\psi, \phi]+\eta \Delta^{\star} \psi \\
\partial_{t} \omega=\rho r\left[\frac{1}{(\rho r)^{2}} \omega, \phi\right]-\rho r\left[\frac{F}{\rho r^{2}}, F\right]-\rho r\left[\frac{1}{\rho r^{2}} \Delta^{\star} \psi, \psi\right]+\nu \Delta_{\rho} \omega \\
\omega=\Delta_{\rho} \phi
\end{array}\right.
$$

Let us the Grad-Shafranov equation [22] for stationary states

$$
\Delta^{\star} \bar{\psi}=-r^{2} \frac{d \bar{p}}{d \bar{\psi}}-\frac{1}{2} \frac{d \bar{F}^{2}}{d \bar{\psi}}
$$

where $\bar{\psi} \mapsto \bar{p}(\bar{\psi})$ and $\bar{\psi} \mapsto \bar{F}(\bar{\psi})$ are two given smooth functions. It is well known that in axisymetric geometry the equilibrium of force between the pressure and the magnetic force holds at $t=0$ if and only if

$$
F=\bar{F}(\bar{\psi}), \quad p=\bar{p}(\bar{\psi}), \text { and } \psi=\bar{\psi} .
$$

The pressure can also be calculated in function of the density in an isentropic model. Therefore one also has

$$
\rho=\bar{\rho}(\bar{\psi}) .
$$

It is necessary to add a source term in the magnetic equation to respect this rest state. In the context of Tokamaks modeling, this is the role of the bootstrap current for which we refer to $[32,33,15]$ and references therein. The system is modified as

$$
\left\{\begin{array}{l}
\partial_{t} \psi=\frac{1}{\rho r}[\psi, \phi]+\eta \Delta^{\star} \psi-\eta \Delta^{\star} \bar{\psi} \\
\partial_{t} \omega=\rho r\left[\frac{1}{(\rho r)^{2}} \omega, \phi\right]-\rho r\left[\frac{F}{\rho r^{2}}, F\right]-\rho r\left[\frac{1}{\rho r^{2}} \Delta^{\star} \psi, \psi\right]+\nu \Delta_{\rho} \omega, \\
\omega=\Delta_{\rho} \phi .
\end{array}\right.
$$

Proposition 18 Solutions of the Grad-Shafranov equation (56-57) are also stationary solutions of (58).

Proof. One has $\left[\frac{\bar{F}}{\rho r^{2}}, \bar{F}\right]=\bar{F} \bar{F}^{\prime}(\bar{\psi})\left[\frac{1}{\rho r^{2}}, \bar{\psi}\right]$. Moreover

$$
\left[\frac{1}{\rho r^{2}} \Delta^{\star} \bar{\psi}, \bar{\psi}\right]=-\left[\frac{1}{\bar{\rho}} \frac{d \bar{p}}{d \bar{\psi}}, \bar{\psi}\right]-\frac{1}{2}\left[\frac{1}{\bar{\rho} r^{2}} \frac{d \bar{F}^{2}}{d \bar{\psi}}, \bar{\psi}\right]=-\bar{F} \bar{F}^{\prime}(\bar{\psi})\left[\frac{1}{\rho r^{2}}, \bar{\psi}\right] .
$$

Therefore the sum of these two terms vanishes, $\left[\frac{F}{\rho r^{2}}, F\right]-\left[\frac{1}{\rho r^{2}} \Delta^{\star} \psi, \psi\right]=0$, which proves the result.

The previous property is an important enhancement of our initial model [15]. Another property is the wellposedness, at least in the weak sense. The proof is based on the result proved in [15]. Indeed the source term $-\left[\frac{F}{\rho r^{2}}, F\right]$ does not modify the structure of the proof, neither the result. 


\subsection{Model with toroidal dependency and constant forcing term}

The next model we consider is (52) with simplifications $\rho_{0}=1$ and $F=F_{0}$ constant. We start from

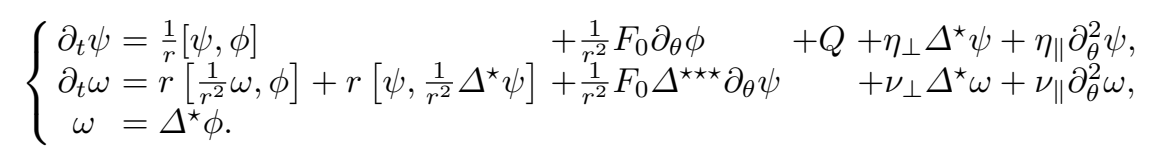

The anisotropic dissipative terms are added to obtain enough dissipativity and are always considered in physical situations. The source term $Q$ has been defined in (52) and can be written in weak form

$$
\int_{\mathcal{D}} \frac{1}{r}\left(\partial_{r} Q \partial_{r} \widetilde{\psi}+\partial_{z} Q \partial_{z} \widetilde{\psi}\right)=2 F_{0} \int_{\mathcal{D}} \frac{1}{r^{4}} \partial_{\theta} \phi \partial_{r} \widetilde{\psi}, \quad \forall \widetilde{\psi} \in H_{0}^{1}(\mathcal{D}) .
$$

We implicitly consider boundary conditions of Dirichlet and Neumann type

$$
\psi=\phi=\frac{\partial \phi}{\partial n}=0 \text { on } \partial \mathcal{D} .
$$

For simplicity the source also satisfies a Dirichlet boundary condition $Q=0$ on $\partial \mathcal{D}$.

\section{Proposition 19}

$$
\begin{gathered}
\frac{1}{2} \frac{d}{d t} \int_{\Omega} \frac{1}{r}\left(\left|\nabla_{r, z} \psi\right|^{2}+\left|\nabla_{r, z} \phi\right|^{2}\right) \\
+\eta_{\perp} \int_{\Omega} \frac{\left|\Delta^{\star} \psi\right|^{2}}{r}+\nu_{\perp} \int_{\Omega} \frac{\left|\Delta^{\star} \phi\right|^{2}}{r}+\eta_{\|} \int_{\Omega} \frac{\left|\partial_{\theta} \nabla_{r, z} \psi\right|^{2}}{r}+\nu_{\|} \int_{\Omega} \frac{\left|\partial_{\theta} \nabla_{r, z} \phi\right|^{2}}{r}=0
\end{gathered}
$$

Proof. Summing the integral form of equations (3) with test functions $-\frac{\Delta^{\star} \psi}{r}$ for the first equation and $-\frac{\phi}{r}$ for the second one, we get the classical part of energy identity for the standard incompressible reduced resistive MHD model, already detailed in [15] for general conditions of density, and some additional terms related to the parallel viscosities $\eta_{\|}, \nu_{\|}$, the forcing term $F_{0}$ and the source term $Q$. We will only deal with the extra terms here.

It is trivial for the parallel diffusion terms through integrations by parts:

$$
\int \eta_{\|} \partial_{\theta}^{2} \psi \frac{\Delta^{\star} \psi}{r}+\int \nu_{\|} \partial_{\theta}^{2} \omega \frac{\phi}{r}=\eta_{\|} \int \frac{\left|\partial_{\theta} \nabla_{r, z} \psi\right|^{2}}{r}+\nu_{\|} \int \frac{\left|\partial_{\theta} \nabla_{r, z} \phi\right|^{2}}{r},
$$

and the forcing and source terms may be treated together as follows:

$$
\begin{aligned}
& \int\left(\frac{1}{r^{2}} F_{0} \partial_{\theta} \phi+Q\right) \frac{\Delta^{\star} \psi}{r}+\int \frac{1}{r^{2}} F_{0} \Delta^{\star \star \star} \partial_{\theta} \psi \frac{\phi}{r} \\
= & \int \frac{1}{r^{3}} F_{0} \partial_{\theta} \phi\left(\Delta^{\star} \psi-\Delta^{\star \star \star} \psi\right)-\frac{\nabla_{r, z} Q \cdot \nabla_{r, z} \psi}{r} \\
= & \int \frac{2}{r^{4}} F_{0} \partial_{\theta} \phi \partial_{r} \psi-\frac{1}{r}\left(\partial_{r} Q \partial_{r} \psi+\partial_{z} Q \partial_{z} \psi\right)
\end{aligned}
$$

since $\Delta^{\star} \psi-\Delta^{\star \star \star} \psi=\left(\Delta \psi-\frac{\partial_{r} \psi}{r}\right)-\left(\Delta \psi-3 \frac{\partial_{r} \psi}{r}\right)=2 \frac{\partial_{r} \psi}{r}$. This contribution vanishes thanks to (60).

Theorem 20 There exists a weak solution to the problem (59)-(60)-(61), related to initial data $\left(\psi_{0}, \phi_{0}\right) \in$ $\left(H_{0}^{1}(\Omega) \cap H^{2}(\Omega)\right) \times H_{0}^{2}(\Omega)$

Proof. We will here rely on the construction of approximate solutions $\left(\psi_{\Delta t}, \phi_{\Delta t}\right)$ detailed in [15] for standard 2-D incompressible reduced resistive MHD model. This construction uses $H^{2}$ regularity of the initial data but this hypothesis can probably be relaxed. In this construction, $\Delta t>0$ is a time step which is used to define a specific and well posed form of the equations, with a splitting in time of the different operators. It is sufficient in the following to consider that $\Delta t>0$ is an abstract regularization parameter and, as usual in the Teman-Lions theory for such systems, the fundamental issue is to show the weak continuity of non linear operators with respect to the norms which are controlled by the energy identity. The main ingredients are provided in the following propositions 21 and 22 . 
Proposition 21 The gradients $\nabla_{r, z} \psi_{\Delta t}$ and $\nabla_{r, z} \phi_{\Delta t}$ are uniformly bounded in $L^{\infty}\left(0, T ; L^{2}(\Omega)\right) \cap L^{2}\left(0, T ; H^{1}(\Omega)\right)$ and by interpolation in $L^{\frac{2}{1-\xi}}\left(0, T ; L^{\frac{6}{2 \xi+1}}(\Omega)\right)$, for all $\left.\xi \in\right] 0,1[$.

Proof. Integrating the energy identity (5) on $[0, T]$, one obtains the uniform a priori bounds:

$$
\begin{gathered}
\nabla_{r, z} \psi_{\Delta t} \in L^{\infty}\left(0, T ; L^{2}(\Omega)\right), \nabla_{r, z} \phi_{\Delta t} \in L^{\infty}\left(0, T ; L^{2}(\Omega)\right) \\
\Delta^{\star} \psi_{\Delta t} \in L^{2}\left(0, T ; L^{2}(\Omega)\right), \Delta^{\star} \phi_{\Delta t} \in L^{2}\left(0, T ; L^{2}(\Omega)\right) \\
\partial_{\theta} \nabla_{r, z} \psi_{\Delta t} \in L^{2}\left(0, T ; L^{2}(\Omega)\right), \partial_{\theta} \nabla_{r, z} \phi_{\Delta t} \in L^{2}\left(0, T ; L^{2}(\Omega)\right)
\end{gathered}
$$

Putting together (63), (64) and (65) we conclude that $\nabla_{r, z} \psi_{\Delta t}$ and $\nabla_{r, z} \phi_{\Delta t}$ are bounded in $L^{\infty}\left(0, T ; L^{2}(\Omega)\right)$ and $L^{2}\left(0, T ; H^{1}(\Omega)\right)$. Next, since $\Omega \subset \mathbb{R}^{3}$, one has the embedding $H^{1}(\Omega) \subset L^{6}(\Omega)$ and then we conclude with the following classical interpolation result

$$
\begin{gathered}
\forall \xi \in(0,1), L^{p_{1}}\left(L^{q_{1}}(\Omega)\right) \cap L^{p_{2}}\left(L^{q_{2}}(\Omega)\right) \subset L^{p}\left(L^{q}(\Omega)\right), \\
\text { with } \frac{1}{p}=\frac{\xi}{p_{1}}+\frac{1-\xi}{p_{2}} \text { and } \frac{1}{q}=\frac{\xi}{q_{1}}+\frac{1-\xi}{q_{2}}
\end{gathered}
$$

applied for $p_{1}=+\infty, q_{1}=2, p_{2}=2, q_{2}=6$.

Proposition $22 \nabla_{r, z} \psi_{\Delta t}$ and $\nabla_{r, z} \phi_{\Delta t}$ are compactly embedded in $L^{2}\left(0, T ; L^{2}(\Omega)\right)$.

Proof. This result will come from the compact injection of $H=\left\{v \in L^{2}\left(0, T ; H^{1}(\Omega)\right) ; \partial_{t} v \in L^{p}(0, T ; B)\right\}$ into $L^{2}\left(0, T ; L^{2}(\Omega)\right)$ for all $1<p<\infty$ and any reflexive Banach space $B$ such that the embedding $L^{2}(\Omega) \subset B$ is continuous. So, let us show that $\nabla_{r, z} \psi_{\Delta t}$ and $\nabla_{r, z} \phi_{\Delta t}$ belong to $H$ for appropriate $p$ and $B$.

First, by Lemma $21, \nabla_{r, z} \psi_{\Delta t}$ and $\nabla_{r, z} \phi_{\Delta t}$ are uniformly bounded in $L^{2}\left(0, T ; H^{1}(\Omega)\right)$.

Next, we get bounds on the time derivatives $\partial_{t} \nabla_{r, z} \psi_{\Delta t}$ and $\partial_{t} \nabla_{r, z} \phi_{\Delta t}$ through the following equations:

$$
\left\{\begin{array}{l}
\partial_{t} \psi_{\Delta t}=\overbrace{\frac{1}{r}\left[\psi_{\Delta t}, \phi_{\Delta t}\right]}^{S_{1}}+\overbrace{\eta_{\perp} \Delta^{\star} \psi_{\Delta t}}^{S_{2}}+\overbrace{\eta_{\|} \partial_{\theta}^{2} \psi_{\Delta t}}^{S_{3}}+\overbrace{\frac{1}{r^{2}} F_{0} \partial_{\theta} \phi_{\Delta t}}^{S_{4}}+Q \\
\partial_{t} \omega_{\Delta t}=\underbrace{r\left[\frac{1}{r^{2}} \omega_{\Delta t}, \phi_{\Delta t}\right]}_{T_{1}}+\underbrace{r\left[\psi_{\Delta t}, \frac{1}{r^{2}} \Delta^{\star} \psi_{\Delta t}\right]}_{T_{2}}+\underbrace{\nu_{\perp} \Delta^{\star} \omega_{\Delta t}}_{T_{3}}+\underbrace{\nu_{\|} \partial_{\theta}^{2} \omega_{\Delta t}}_{T_{4}}+\underbrace{\frac{1}{r^{2}} F_{0} \Delta^{\star \star \star} \partial_{\theta} \psi_{\Delta t}}_{T_{5}}
\end{array}\right.
$$

Notice that $r, F_{0}$ and diffusion coefficients are bounded from above and below and thus play no role here. We also remark that the Poisson brackets can be written in the following different forms: $[a, b]=\nabla_{r, z} a$. $\nabla_{r, z}^{\perp} b=\operatorname{div}_{r, z}\left(a \nabla_{r, z}^{\perp} b\right)$ where $\nabla_{r, z}^{\perp} b=\left(-\partial_{z} b, 0, \partial_{r} b\right)$. By Lemma 21, one has $\nabla_{r, z} \psi_{\Delta t} \in L^{\infty}\left(0, T ; L^{2}(\Omega)\right)$, $\nabla_{r, z}^{\perp} \phi_{\Delta t} \in L^{2}\left(0, T ; L^{6}(\Omega)\right)$, and

$$
S_{1}=\frac{1}{r} \nabla_{r, z} \psi_{\Delta t} \cdot \nabla_{r, z}^{\perp} \phi_{\Delta t} \text { belong to } L^{2}\left(0, T ; L^{s}(\Omega)\right), \text { for all } 1<s \leq \frac{3}{2} .
$$

By Lemma 21 and (64), we get $\omega_{\Delta t} \cdot \nabla^{\perp} \phi_{\Delta t} \in L^{2}\left(L^{2}(\Omega)\right) \times L^{\frac{2}{1-\xi}}\left(0, T ; L^{\frac{6}{2 \xi+1}}(\Omega)\right)$ for all $\left.\xi \in\right] 0,1[$. This leads to $\omega_{\Delta t} \cdot \nabla^{\perp} \phi_{\Delta t} \in L^{\frac{2}{2-\xi}}\left(0, T ; L^{\frac{3}{\xi+2}}(\Omega)\right)$ for all $\left.\xi \in\right] 0,1[$ and then

$$
\left.T_{1}=r \operatorname{div}_{r, z}\left(\frac{1}{r^{2}} \omega_{\Delta t} \cdot \nabla^{\perp} \phi_{\Delta t}\right) \in L^{\frac{2}{2-\xi}}\left(0, T ; W^{-1, \frac{3}{\xi+2}}(\Omega)\right), \text { for all } \xi \in\right] 0,1[.
$$

Analogously, by Lemma 21 and (64),

$$
\left.T_{2}=r \operatorname{div}_{r, z}\left(\frac{1}{r^{2}} \Delta^{\star} \psi_{\Delta t} \cdot \nabla^{\perp} \psi_{\Delta t}\right) \in L^{\frac{2}{2-\xi}}\left(0, T ; W^{-1, \frac{3}{\xi+2}}(\Omega)\right) \text {, for all } \xi \in\right] 0,1[.
$$

All the remaining linear terms belong to $L^{2}\left(0, T ; H^{-2}(\Omega)\right)$ and this is enough to conclude that

$$
\partial_{t} \nabla_{r, z} \psi_{\Delta t} \text { and } \partial_{t} \nabla_{r, z} \phi_{\Delta t} \text { belong to } L^{p}(0, T ; B) \text {, with } B=H^{-3}(\Omega)
$$

and $p=\frac{2}{2-\xi}$ for any $\left.\xi \in\right] 0,1[$. 


\subsection{Remarks on the role of the correction term}

It is instructing to analyze the role of the correction term $Q$ with respect to the well-posedness of the final reduced models. Our claim is that $Q$ is related to the spectral stability of the partial differential operator with respect to the angular variable $\theta$. More precisely the model with the $Q$ might be linearly ill-posed in the limit of vanishing resistivity and viscosity.

To understand this fact we simplify (59-60). Retaining only the derivatives with respect to $\theta$, and taking into account that $\Delta^{\star}=\partial_{r r}-\frac{1}{r} \partial_{r}$ and $\Delta^{\star}=\partial_{r r}-\frac{3}{r} \partial_{r}$, one gets the set of linear equations

$$
\left\{\begin{array}{l}
\partial_{t} \psi=\frac{F_{0}}{r^{2}} \partial_{\theta} \phi+Q, \\
\partial_{t} \omega=\frac{F_{0}}{r^{2}}\left(\partial_{r r}-\frac{3}{r} \partial_{r}\right) \partial_{\theta} \psi, \\
\omega=\left(\partial_{r r}-\frac{1}{r} \partial_{r}\right) \phi, \\
\int \frac{1}{r} \partial_{r} Q \partial_{r} \widetilde{\psi}^{2}=2 F_{0} \int \frac{1}{r^{4}} \partial_{\theta} \phi \partial_{r} \widetilde{\psi}, \forall \widetilde{\psi} \in Z .
\end{array}\right.
$$

It is assumed that $Z$ is a convenient space of trial function such that at least $H_{0}^{1}(I) \subset Z$. In the following we systematically disregard boundary conditions since we are interested in the behavior of the correction term $Q$ inside the domain of study.

We therefore consider for simplicity that the last weak formulation of (66) is replaced by

$$
\partial_{r} Q=\frac{2 F_{0}}{r^{3}} \partial_{\theta} \phi
$$

Next we replace any function with its Fourier transform in the direction $\theta$. One obtains the system $(n \in \mathbb{Z})$

$$
\left\{\begin{array}{l}
\partial_{t} \widehat{\psi}_{n}=\mathbf{i} n \frac{F_{0}}{r^{2}} \widehat{\phi}_{n}+\mathbf{i} n F_{0} \widehat{q}_{n}, \\
\partial_{t} \widehat{\omega}_{n}=\mathbf{i} n \frac{F_{0}}{r^{2}}\left(\partial_{r r}-\frac{3}{r} \partial_{r}\right) \widehat{\psi}_{n} \\
\widehat{\omega}_{n}=\left(\partial_{r r}-\frac{1}{r} \partial_{r}\right) \widehat{\phi}_{n} \\
\partial_{r} \widehat{q}_{n}=\frac{2}{r^{3}} \widehat{\phi}_{n}
\end{array}\right.
$$

The spectral stability amounts to consider a dependence $\widehat{f}(t, r)=e^{\lambda t} \widetilde{f}(r)$. One obtains the system

$$
\left\{\begin{array}{l}
\lambda \widetilde{\psi}_{n}=\mathbf{i} n F_{0}\left(\frac{1}{r^{2}} \widetilde{\phi}_{n}+\widetilde{q}_{n}\right), \\
\lambda \widetilde{\omega}_{n}=\mathbf{i} n \frac{F_{0}}{r^{2}}\left(\partial_{r r}-\frac{3}{r} \partial_{r}\right) \widetilde{\psi}_{n}, \\
\widetilde{\omega}_{n}=\left(\partial_{r r}-\frac{1}{r} \partial_{r}\right) \widetilde{\phi}_{n}=r \partial_{r}\left(\frac{1}{r} \partial_{r} \widetilde{\phi}_{n}\right), \\
\partial_{r} \widetilde{q}_{n}=\frac{2}{r^{3}} \widetilde{\phi}_{n} .
\end{array}\right.
$$

The analogous of the energy identity is as follows. First

$$
\begin{aligned}
& \lambda \int \frac{\left|\widetilde{\psi}_{n}^{\prime}\right|^{2}+\left|\widetilde{\phi}_{n}^{\prime}\right|^{2}}{r} d r=-\lambda \int \frac{1}{r} \widetilde{\psi}_{n}\left(\partial_{r r}-\frac{1}{r} \partial_{r}\right) \overline{\widetilde{\psi}_{n}}+\frac{1}{r} \omega_{n} \overline{\widetilde{\phi}_{n}} \\
& =-\mathbf{i} n F_{0} \int \frac{1}{r}\left(\frac{1}{r^{2}} \widetilde{\phi}_{n}+\widetilde{q}_{n}\right)\left(\partial_{r r} \overline{\widetilde{\psi}_{n}}-\frac{1}{r} \partial_{r} \widetilde{\widetilde{\psi}_{n}}\right)+\frac{1}{r}\left(\frac{1}{r^{2}}\left(\partial_{r r}-\frac{3}{r} \partial_{r}\right) \widetilde{\psi}_{n}\right) \widetilde{\widetilde{\phi}_{n}} \\
& =-\mathbf{i} n F_{0} \underbrace{\int \frac{1}{r} \widetilde{q}_{n}\left(\partial_{r r} \overline{\widetilde{\psi}_{n}}-\frac{1}{r} \partial_{r} \overline{\widetilde{\psi}_{n}}\right)-\frac{1}{r^{3}}{\widetilde{\phi_{n}}}_{n} \frac{2}{r} \partial_{r} \widetilde{\psi}_{n}}_{=A} \\
& -\mathbf{i} n F_{0} \underbrace{\int \frac{1}{r^{3}}\left(\widetilde{\phi}_{n}\left(\partial_{r r} \overline{\widetilde{\psi}_{n}}-\frac{1}{r} \partial_{r} \overline{\widetilde{\psi}_{n}}\right)+\left(\partial_{r r} \widetilde{\psi}_{n}-\frac{1}{r} \partial_{r} \widetilde{\psi}_{n}\right) \overline{\widetilde{\phi}_{n}}\right)}_{=B} .
\end{aligned}
$$

The second term is real $B \in \mathbb{R}$. An integration by parts (still forgetting about the boundary terms), together with the definition of the correction term $\widetilde{q}_{n}$ gives

$$
A=-\int \frac{1}{r} \partial_{r} \widetilde{q}_{n} \partial_{r} \widetilde{\widetilde{\psi}_{n}}-\frac{2}{r^{4}} \widetilde{\widetilde{\phi}_{n}} \partial_{r} \widetilde{\psi}_{n}=-\int \frac{2}{r^{4}}\left(\widetilde{\phi}_{n} \partial_{r} \widetilde{\widetilde{\psi}_{n}}+\widetilde{\widetilde{\phi}_{n}} \partial_{r} \widetilde{\psi}_{n}\right) \in \mathbb{R}
$$

Thus, it yields

$$
\lambda=-\mathbf{i} n F_{0}(A+B)\left(\int \frac{\left|\widetilde{\psi}_{n}^{\prime}\right|^{2}+\left|\widetilde{\phi}_{n}^{\prime}\right|^{2}}{r} d r\right)^{-1} \in \mathbf{i} \mathbb{R}
$$


which in turns corresponds to the linear of spectral stability of (68) or (69). Once again this analysis is qualitative and valid away from the boundary.

If one forgets the correction, the algebra is almost the same except that now

$$
A=-\int \frac{2}{r^{4}} \widetilde{\phi}_{n} \partial_{r} \widetilde{\psi}_{n}
$$

The first equation of (69) is also modified $\lambda \widetilde{\psi}_{n}=\mathbf{i} n \frac{F_{0}}{r^{2}} \widetilde{\phi}_{n}$. Now $A$ has no reason a priori to be real. If $A$ admits an imaginary part, the model might be linear instable. More studies are needed to confirm, or not, this hypothesis. In any case, we conclude that the correction term, which is related to the metric, that is to the curvature of the domain (a torus for Tokamaks), guarantees the well-posedness.

It must be noted that the correction term is not present in all models in Tokamak geometry used for numerical simulation that we know about in the literature, see for example $[23,26,28,37,40,42]$.

\section{Linearized stability of the hierarchy}

We end this work with some considerations on the stability of the hierarchy of abstract linearized equations (17). Let us start with a reference rest state $U_{0}^{\prime}$, that is $V_{0}^{\prime}=\nabla_{U_{0}^{\prime}} S \in \mathcal{K}$, and

$$
\nabla \cdot f\left(U_{0}^{\prime}\right)=0
$$

Assuming that boundary conditions do not yield additional perturbation the function $U(x, t)=U_{0}^{\prime}(x)$ is a solution of (15). Notice that constant (in space) states are rest states of course, but constant states might not belong to $\mathcal{K}$. This is in practice the case for equilibrium solutions in Tokamaks since physical rest states satisfy the Grad-Shafranov equation (55). The study of the dynamo effect in astrophysics yield similar questions, we refer to $[20,49]$.

So we add a perturbation to the initial condition $U_{\varepsilon}(0)=\widehat{U_{\varepsilon}}$, that is

$$
\widehat{U_{\varepsilon}}=U_{0}^{\prime}+\varepsilon U_{1}+o(\varepsilon) \text {. }
$$

Let us now consider the solution with initial condition $U_{\varepsilon}(0)$. A natural question is to determine the evolution of the perturbation, at least in the linear regime.

By subtraction one gets

$$
\int_{\Omega}\left[\left(\partial_{t} \frac{U_{\varepsilon}-U_{0}^{\prime}}{\varepsilon}, Z\right)+\left(\nabla \frac{f\left(U_{\varepsilon}\right)-f\left(U_{0}^{\prime}\right)}{\varepsilon}, Z\right)\right] d v=0, \quad \forall Z \in d \mathcal{K} .
$$

The linearized adjoint unknown

$$
V_{\varepsilon}=V_{0}^{\prime}+\varepsilon V_{1}+o(\varepsilon)
$$

A Taylor expansion yields

and

$$
\frac{U_{\varepsilon}-U_{0}^{\prime}}{\varepsilon}=\left(\nabla_{V_{0}^{\prime}} U\right) V_{1}+o(1)
$$

$$
\frac{f\left(U_{\varepsilon}\right)-f\left(U_{0}^{\prime}\right)}{\varepsilon}=\left(\nabla_{V_{0}^{\prime}} f(U)\right) V_{1}+o(1)
$$

Take care that $\nabla_{V_{0}^{\prime}} f(U) \in \mathbb{R}^{d \times n \times n}$ is a tensor. One has the classical relations

$$
\nabla_{V} U=d_{V}^{2} S^{\star}=\left(d_{U(V)}^{2} S\right)^{-1} \text { and } \nabla_{V} f(U)=d_{V}^{2} F^{\star}
$$

where the Legendre (resp. polar) transform of $S$ (resp. $F$ ) is

$$
S^{\star}(V)=(V, U(V))-S(U(V)) \quad\left(\text { resp. } F^{\star}(V)=(V, f(U(V)))-F(U(V))\right) .
$$

Let us define $A_{0}(x)=d_{V_{0}^{\prime}}^{2} S^{\star}$ and $A_{1}(x)=d_{V_{0}^{\prime}}^{2} F^{\star}$ which are a priori dependent of the space variable $x$ since $V_{0}^{\prime} \in \mathcal{K}$ also depends on the space variable. The formal limit of (72) yields the linearized formulation

$$
\int_{\Omega}\left[\partial_{t}\left(A_{0} V_{1}\right)+\nabla \cdot\left(A_{1} V_{1}\right), Z\right] d v=0, \quad \forall Z \in d \mathcal{K} .
$$

Since $V_{\varepsilon}, V_{0}^{\prime} \in \mathcal{K}$, one can pass to the limit in $V_{1}=\lim _{\varepsilon} \frac{V_{\varepsilon}-V_{0}^{\prime}}{\varepsilon}$ to obtain

$$
V_{1} \in d \mathcal{K} \text {. }
$$


Proposition 23 (Linearized stability) Solutions of the linearized formulation (74-75) satisfy

$$
\frac{d}{d t} \int_{\Omega}\left(V_{1}, A_{0} V_{1}\right) d v=\int_{\Omega}\left(\left(\nabla \cdot A_{1}\right) V_{1}, V_{1}\right) d v+\text { b.c. }
$$

Proof. The proof proceeds by taking $Z=V_{1}$ in (74) and integration in space using the identity

$$
\left(\nabla \cdot\left(A_{1} V_{1}\right), V_{1}\right)=\nabla \cdot \frac{\left(\left(A_{1} V_{1}\right), V_{1}\right)}{2}-\left(\left(\nabla \cdot A_{1}\right) V_{1}, V_{1}\right) .
$$

Let us assume, for simplicity, that there exists $\alpha>0$ such that $d_{V_{0}^{\prime}}^{2} S^{\star} \geq \alpha \mathbf{I}>0$. It yields $\alpha$-convexity: in this case it is possible in theory to use (76) with a Gronwall lemma to obtain a control of $V_{1}(t)$ in some adapted $L^{2}$ based norm. This is why the identity is fundamentally a stability result. It establishes, for hyperbolic compatible models, the linearized stability of rest states.

\subsection{An abstract comparison principle}

It is well known that the method of moment is endowed with a comparison principle $[9,4,5,13]$ for the eigenvalues of the Jacobian matrix. It is therefore natural to question about a generalization of this principle to our problem. We found the following generalization which can be interpreted as an evaluation of the growth rate of modes in the linear regime.

It is convenient for mathematical development to insure a minimal amount of compactness since we deal with functions. This is why we add dissipation to the initial model and consider

$$
\left\{\begin{array}{l}
\int_{\Omega}\left[\partial_{t} U+\nabla \cdot f(U)-\nu \Delta\left(U-U_{0}^{\prime}\right), Z\right] d v=0, \forall Z \in d \mathcal{K}, \\
V \in \mathcal{K} .
\end{array}\right.
$$

Notice that $U_{0}^{\prime}$ is incorporated in the dissipation term to respect the fact that $U_{0}^{\prime}$ is a rest state. In the context of the modeling of Tokamaks, the so-called bootstrap current $[32,33,15]$ is exactly of this nature. The dissipation right hand side can be replaced by a more general dissipative operator $\nabla \cdot\left(D(U) \nabla\left(U-U_{0}^{\prime}\right)\right)$ where $D(U)=D(U)^{t}$ is a given positive dissipation tensor compatible with the entropy principle $[9,4,5]$. For the sake of simplicity we consider here only the simplified dissipative term $\Delta\left(U-U_{0}^{\prime}\right)$ with a viscous coefficient $\nu>0$. From now on we only comment the consequences of the introduction of this very specific dissipation term.

The linearized equations (71-75) write now

$$
\int_{\Omega}\left[\partial_{t}\left(A_{0} V_{1}\right)+\nabla \cdot\left(A_{1} V_{1}\right)-\nu \nabla \cdot\left(A_{0} \nabla V_{1}\right), Z\right] d v=0, \quad \forall Z \in d \mathcal{K},
$$

still with $V_{1} \in d \mathcal{K}$. Taking $Z=V_{1}$ and assuming that all boundary terms vanish, one obtains by integration by parts the generalization of $(76)$

$$
\frac{d}{d t} \int_{\Omega}\left(V_{1}, A_{0} V_{1}\right) d v=\int_{\Omega}\left(\left(\nabla \cdot A_{1}\right) V_{1}, V_{1}\right) d v-2 \nu \int_{\Omega}\left(A_{0} \nabla V_{1}: \nabla V_{1}\right) d v
$$

Let us define the closed subspace of $H_{0}^{1}(\Omega)^{n}$

$$
Y(d \mathcal{K})=\overline{d \mathcal{K} \cap H_{0}^{1}(\Omega)^{n}}
$$

and the real number $\lambda\left(V_{0}^{\prime}, d \mathcal{K}\right) \in \mathbb{R}$

$$
\lambda\left(V_{0}^{\prime}, d \mathcal{K}\right)=\sup _{V_{1} \in Y(\mathcal{K})} \frac{\int_{\Omega}\left(\left(\nabla \cdot A_{1}\right) V_{1}, V_{1}\right) d v-2 \nu \int_{\Omega}\left(A_{0} \nabla V_{1}: \nabla V_{1}\right) d v}{\int_{\Omega}\left(V_{1}, A_{0} V_{1}\right) d v} .
$$

We make usual assumptions on the boundedness [1] of the symmetric matrices $A_{0}(x)$ and $\nabla \cdot A_{1}(x)$

$$
0<\alpha_{-} \leq A_{0}, \quad \text { and }\left\|A_{0}\right\|_{L^{\infty}(\Omega)^{n \times n}}+\left\|\nabla \cdot A_{1}\right\|_{L^{\infty}(\Omega)^{n \times n}}<\infty .
$$

Proposition 24 Under the previous assumptions, $\lambda\left(V_{0}^{\prime}, d \mathcal{K}\right)$ is well defined by (78). 
Proof. Let us take a sufficiently large number $l>0$ and set $C=l A_{0}-A_{1}$ which is non negative: $C \geq 0$. Then

$$
\lambda\left(V_{0}^{\prime}, d \mathcal{K}\right)-l=\sup _{W \in Y(\mathcal{K})} \frac{-(C W, W)-\left(A_{0} \nabla W: \nabla W\right)}{\left(A_{0} W, W\right)} .
$$

So it is equivalent to say that

$$
\left(\lambda\left(V_{0}^{\prime}, d \mathcal{K}\right)-l\right)^{-1}=\sup _{W \in Y(\mathcal{K})} \frac{\left(A_{0} W, W\right)}{(C W, W)+\left(A_{0} \nabla W: \nabla W\right)}
$$

The denominator is an equivalent norm in $H_{0}^{1}(\Omega)^{n}$. So compactness properties of $Y(\mathcal{K})$ implies that the sup is also a max [1].

Set $a(t)=\left(\int_{\Omega}\left(V_{1}, A_{0} V_{1}\right) d v\right)(t)$. The relation (77) yields

$$
a^{\prime}(t) \leq \lambda\left(V_{0}^{\prime}, d \mathcal{K}\right) a(t) \Rightarrow a(t)^{\frac{1}{2}} \leq e^{\frac{1}{2} \lambda\left(V_{0}^{\prime}, d \mathcal{K}\right) t} a(0)^{\frac{1}{2}} .
$$

This is why $\frac{1}{2} \lambda\left(V_{0}^{\prime}, d \mathcal{K}\right)$ is an upper bound of the growth rate of modes in the linear regime. If by chance the rest state is such that $\lambda\left(V_{0}^{\prime}, d \mathcal{K}\right)=0$, then this rest state is endowed with very strong stability properties since the exponential growth is actually constant in time. An even better situation is of course if $\lambda\left(V_{0}^{\prime}, d \mathcal{K}\right)<0$. The next result states that $\lambda\left(V_{0}^{\prime}, d \mathcal{K}\right)$ is a lower bound of the same quantity for the initial model.

Theorem 25 Assume $d \mathcal{K}_{1} \subset d \mathcal{K}_{2} \subset H_{0}^{1}(\Omega)^{n}$. Then

$$
\lambda\left(V_{0}^{\prime}, d \mathcal{K}_{1}\right) \leq \lambda\left(V_{0}^{\prime}, d \mathcal{K}_{2}\right) \leq \lambda\left(V_{0}^{\prime}, H_{0}^{1}(\Omega)^{n}\right) .
$$

Proof. This is immediate from the definition (78).

An interpretation of this abstract comparison principle is the following. Let us assume that one is interested by rest states $V_{0}^{\prime}$ which are also stable with respect to small perturbation. In such a situation, it is worthwhile to seek for states $V_{0}^{\prime}$ such that $\lambda\left(V_{0}^{\prime}, H_{0}^{1}(\Omega)^{n}\right)$ is the smallest as possible, which in turns implies boundedness and control of $\lambda\left(V_{0}^{\prime}, d \mathcal{K}\right)$ for any $\mathcal{K}$. In our mind such an interpretation could be indicative of the stability of the state $V_{0}^{\prime}$. With this respect instability of one single reduced model may be a good indication of the instability of the initial model before reduction.

Acknowledgments Authors acknowledge the support of ANR under contract ANR-12-BS01-0006-01. Moreover this work was carried out within the framework of the European Fusion Development Agreement and the French Research Federation for Fusion Studies. It is supported by the European Communities under the contract of Association between Euratom and CEA. The views and opinions expressed herein do not necessarily reflect those of the European Commission.

\section{References}

1. G. Allaire, Numerical Analysis and Optimization: An Introduction to Mathematical Modelling and Numerical Simulation (Numerical Mathematics and Scientific Computation series), Oxford University Press (2007).

2. D. Biskamp, Nonlinear Magnetohydrodynamics, Cambridge University Press (1992).

3. J. Blum, Numerical simulation and optimal control in plasma physics, with application to Tokamaks, Wiley/GauthierVillard series in modern applied mathematics, 1989.

4. G. Boillat and T. ruggeri, Hyperbolic principal subsystems: entropy convexity and subcharacteristic conditions. Arch. rational Mech. Anal. 137 (1997), no. 4, 305-320.

5. Boillat, G., Ruggeri, T.: Moment equations in the kinetic theory of gases and wave velocities. Contin. Mech. Thermodyn. 9 (1997), 205-212.

6. J. Breslau, N. Ferraro, S. Jardin, Some properties of the M3D-C1 form of the 3D magnetohydrodynamics equations, Phys. Plasmas, 16, 2009.

7. L. Chacon, D.A. Knoll, J.M. Finn, An Implicit, Nonlinear Reduced Resistive MHD Solver, Journal of Computational Physics, Volume 178, Issue 1, 1, Pages 1536, 2002.

8. F. Chen, Introduction to plasma physics and controlled fusion, Springer New-York, 1984.

9. G.Q. Chen and C.D. Levermore and T.P. Liu, Hyperbolic conservation laws with stiff relaxation and entropy, Communications in Pure an Applied Mathematics, 787-830, 1994.

10. O. Czarny, G. Huysmans, Bézier surfaces and finite elements for MHD simulations, JCP 227, 7423-7445 (2008).

11. O. Czarny, G. Huysmans, MHD stability in X-point geometry: simulation of ELMs, Nucl. Fusion 47, 659-666 (2007).

12. J.L. Delcroix and A. Bers, Physique des plasmas, 1994, CNRS.

13. B. Després, A geometrical approach to nonconservative shocks and elastoplastic shocks. Arch. ration. Mech. Anal., $186,275308,2007$. 
14. B. Després, S.K. Malapaka and R. Sart, Numerical simulations of a new Generalized reduced resistive MHD model for current hole Plasmas, in preparation.

15. B. Després, R. Sart, reduced resistive MHD in Tokamaks with general density, M2AN online 2012.

16. P. Dirac, Lecture notes on quantum physics, reprint 2001, Dover.

17. J.F. Drake and T.M. Antonsen, Nonlinear reduced fluid equations for toroidal plasmas, Phys., Fluids, 27, 898-908, 1984.

18. J. Freidberg, Plasma physics and energy fusion, cambridge, 2007.

19. T. Fujita, Tokamak equilibria with nearly zero central current: the current hole (review article), Nucl. Fusion 50, (2010).

20. A.D. Gilbert, Dynamo theory. Hanbook of mathematical fluid dynamics, 2(1): 355-441, 2003.

21. H. Grad, On the kinetic theory of rarefied gases. Comm. Pure Appl. Math., 2:331, 1949.

22. H. Grad and H. Rubin, Hydromagnetic Equilibria and Force-Free Fields, Journal of Nuclear Energy (1954), vol. 7, no. 3-4, pp. 284-285, 1958.

23. M. Hölzl, S. Günter, R.P. Wenninger, W.-C. Mller, G.T.A. Huysmans, K. Lackner, I. Krebs, and the ASDEX Upgrade Team, Reduced-MHD Simulations of Toroidally and Poloidally Localized ELMs, online arxiv http://arxiv.org/abs/1201.5765, 2012.

24. S. Jardin, Computational Methods in Plasma Physics (Chapman \& Hall/CRC Computational Science), 2010.

25. S.E. Kruger, Generalized reduced magnetohydrodynamic equations, Master report, University of Wisconsin, Madison, 1999.

26. S.E. Kruger, C.C. Hegna and J.D. Callen, Generalized reduced magnetohydrodynamic equations, Physics of plasmas, 5 (12), 1998.

27. P.D. Lax, Hyperbolic systems of conservation laws and the theory of shock waves, SIAM, Philadelphia, 1973.

28. E. Lazzaro, L. Comisso and M. Del Pra, Nonlinear and Diamagnetic Effects in a Neoclassical Model of Magnetic Reconnection, AIP Conf. Proc. 1392, pp. 45-54, IFP-CNR-Chalmers Workshop on nonlinear phenomena in fusion plasmas, 2011.

29. J.-L. Lions, Quelques méthodes de résolution des problèmes aux limites non linéaires, Etudes Mathématiques, Dunod (1969).

30. P.-L. Lions, Mathematical topics in fluid mechanics, Vol 1. Incompressible models. Oxford Science Publication, Oxford, (1996).

31. P.-L. Lions, Mathematical topics in fluid mechanics, Vol 2. Compressible models. Oxford Science Publication, Oxford, (1998).

32. H. Lütjens and J.-F. Luciani, The XTOR code for nonlinear 3D simulations of MHD instabilities in tokamak plasmas, Journal of Computational Physics, Volume 227, Issue 14, 1, 6944-6966, (2008).

33. H. Lütjens and J.-F. Luciani, XTOR-2F: A fully implicit NewtonKrylov solver applied to nonlinear 3D extended MHD in tokamaks, Journal of Computational Physics, Volume 229, Issue 21, 8130-8143, (2010).

34. I. Muller and T. Ruggeri: Rational Extended Thermodynamics. 2nd ed. Springer Tracts of Natural Philosophy Vol. 37. Springer New York (1998

35. B. Nkonga, Numerical approximations: Finite elements for MHD modeling, 4th AE-Fusion Summer School on Numerical Modelling for Fusion, Max-Planck-Institut fur Plasmaphysik, 2012.

36. K. Obrejan, Master report, 2012.

37. B. Philip, M. Pernice, and L. Chacon, Solution of Reduced Resistive Magnetohydrodynamics using Implicit Adaptive Mesh Refinement, Proceedings of the 16th International Conference on Domain Decomposition Methods, 2007.

38. G. Poette, B. Després and D. Lucor, Uncertainty quantification for systems of conservation laws Original, Journal of Computational Physics, Volume 228, Issue 7, 20 April 2009, Pages 2443-2467.

39. G. Poette, B. Després and D. Lucor, Review of robust uncertainty propagation in systems of conservation laws with the entropy closure method, to appear in LNSCE 2012.

40. S. Pamela and G.T.A. Huysmans, Equilibrium Flows in Non-linear MHD Simulations of X-point Plasmas, Theory of fusion plasmas, AIP Conference Proceedings, Volume 1069, pp. 318-324 (2008).

41. A. Pataki, A.J. Cerfon, J.P. Freidberg, L. Greengard and M. ONeil, A fast, high-order solver for the Grad-Shafranov equation, arxiv online $1210.2113 \mathrm{v} 1,2012$.

42. F. Romanelli, F. Zonca, A reduced set of equations for resistive fluid turbulence in toroidal systems, Plasma Physics and Controlled Fusion, Vol 31. No.9, pp. 1365 to 1379, 1989.

43. M.N. Rosenbluth, D.A. Monticello, H.R. Strauss, R.B. White, Dynamics of high $\beta$ plasmas, Physics of Fluids 19, 1987- (1976).

44. H.R. Strauss, Dynamics of high $\beta$ plasmas, Physics of Fluids 20, 1354-1360 (1977).

45. R. Sart, B. Després, Derivation of hierarchies of reduced MHD models in Tokamak geometry, arxiv preprint 2013.

46. H.R. Strauss, Nonlinear Three-Dimensional Magnetohydrodynamics of Noncircular Tokamaks, Physics of Fluids 19, 134-140 (1976).

47. H.R. Strauss, Reduced MHD for mirror machines, 1982 Nucl. Fusion 22893.

48. R. Temam, Navier-Stokes Equations, Theory and Numerical Analysis. North-Holland. (1979)

49. M.M. Vishik, S. Friedlander, Dynamo theory methods for hydrodynamic instability. Journal de mathématiques pures et appliquées, 72(2): 145-180, 1993.

50. G. Vlad, G. Bracco, P. Buratti, Scaling of the sawtooth repetition time from simulations with reduced MHD equations, and comparison with experiments in the Frascati Tokamak, 1991 Nucl. Fusion 311536. 